\title{
SWR1C catalyzes H2A.Z deposition by coupling ATPase activity to the nucleosome acidic patch
}

\author{
Nathan Gioacchini ${ }^{1,2}$ and Craig L. Peterson ${ }^{1,3}$
}

${ }^{1}$ Program in Molecular Medicine, University of Massachusetts Medical School, Worcester, MA, 01605

${ }^{2}$ Interdisciplinary Graduate Program, University of Massachusetts Medical School, Worcester, MA, 01605

${ }^{3}$ Corresponding author. Email:craig.peterson@umassmed.edu; phone 508-856-5858

Key words: Chromatin remodeling, transcription, nucleosome, H2A.Z, SWR1C

Running header: SWR1C activity requires the nucleosome acidic patch 


\section{Summary}

The SWR1C chromatin remodeling enzyme catalyzes the ATP-dependent exchange of nucleosomal histone $\mathrm{H} 2 \mathrm{~A}$ for the histone variant H2A.Z, a key variant involved in a multitude of nuclear functions. How the 14-subunit SWR1C engages the nucleosomal substrate remains largely unknown. Numerous studies on the ISWI, CHD1, and SWI/SNF families of chromatin remodeling enzymes have demonstrated an essential role for the nucleosomal acidic patch for remodeling activity, however a role for this nucleosomal epitope in nucleosome editing by SWR1C has not been tested. Here, we employ a variety of biochemical assays to demonstrate an essential role for the nucleosomal acidic patch in the H2A.Z exchange reaction. Nucleosomes lacking acidic patch residues retain the ability to stimulate the ATPase activity of SWR1C, implicating a role in coupling the energy of ATP hydrolysis to $\mathrm{H} 2 \mathrm{~A} / \mathrm{H} 2 \mathrm{~B}$ dimer eviction. A conserved arginine-rich region within the Swc5 subunit is identified that interacts with the acidic patch and is found to be essential for dimer exchange activity. Together these findings provide new insights into how SWR1C engages its nucleosomal substrate to promote efficient $\mathrm{H} 2 \mathrm{~A} . \mathrm{Z}$ deposition.

\section{Introduction}

Eukaryotic genomes regulate access to information stored in their genetic code through the dynamic nucleoprotein structure known as chromatin. The basic unit of chromatin, the nucleosome, sequesters $\sim 147$ base pairs (bp) of DNA around a histone octamer consisting of a H3-H4 tetramer flanked by two H2A-H2B hetero-dimers (1). Rather than a static structure, chromatin is highly dynamic, due to the coordinated impact of histone post translational modifications, ATP-dependent chromatin remodeling enzymes, and the incorporation of histone variants (2). Disruption of these processes can have deleterious consequences for all nuclear events, including transcription, DNA replication, and genome stability pathways.

H2A.Z, a variant of the core histone H2A, is enriched within nucleosomes adjacent to genes transcribed by RNA polymerase II, replication origins, centromeres, and at sites of DNA double strand break repair (3-7). In yeast, H2A.Z enrichment correlates with higher rates of nucleosome turnover that facilitates transcription, as well as antagonizing the spread of heterochromatic regions from telomeres and the silent mating type loci (8-10). While yeast $\mathrm{H} 2 \mathrm{~A} . \mathrm{Z}$ is not essential for viability, H2A.Z is essential in metazoans where it is associated with both transcriptional activation and repression (11). For instance, in embryonic stem cells, deposition and function of $\mathrm{H} 2 \mathrm{~A} . \mathrm{Z}$ is interdependent with the polycomb repressive complex 2 (PRC2), and loss of either H2A.Z or PRC2 leads to transcriptional de-repression of a host genes and a subsequent failure in lineage commitment (12).

The site-specific deposition of H2A.Z is catalyzed by ATP-dependent chromatin remodeling enzymes related to the 14-subunit, yeast SWR1C complex (13, 14). Mammals contain two enzymes related to SWR1C, the SRCAP and p400/Tip60 complexes, and subunits of the mammalian enzymes are highly conserved with those of SWR1C $(15,16)$. Each of these enzymes are members of the larger INO80 subfamily of chromatin remodeling enzymes, distinguished by a large insertion domain between the two ATPase lobes of the catalytic 
ATPases (Swr1 in SWR1C) $(2,17,18)$. This insertion domain serves as a docking site for several key subunits of SWR1C-like complexes, including the Rvb1/Rvb2 heterohexameric ring (RUVB1/RUVB2 in humans) which acts as a further scaffold for organizing additional subunits involved in nucleosome recognition $(19,20)$. In addition to Rvb1/Rvb2, previous studies have demonstrated that the deposition of H2A.Z by SWR1C requires many subunits, including Swc2, Swc4, Yaf9, and Swc5 (19, 21). Swc2 and its mammalian ortholog, Y1-1, promotes nucleosome binding and assists SWR1C in the recognition of nucleosome free regions adjacent to gene promoter regions $(19,22,23)$. Swc4, DMAP1 in mammals, is also part of the yeast NuA4 histone acetyltransferase complex, and it also appears to promote DNA and histone binding (24, 25). Swc5, Cfdp1 in mammals, is essential for the ATPase activity of SWR1C, though it does not impact binding of the enzyme to nucleosomes or to the free H2A.Z/H2B co-substrate $(19,26)$. The key role for Swc5 requires a conserved C-terminal domain, termed BCNT, but how Swc5 promotes SWR1C activity is largely unknown (27).

The subunit complexity of chromatin remodelers varies from the single subunit, yeast CHD1, to multi-subunit 1MDa enzymes such as RSC, SWI/SNF, INO80C, and SWR1C. Despite differences in function and complexity, recent studies have shown that the activity of many remodelers require that they engage a solvent exposed, acidic surface on the nucleosome (28-30). This "acidic patch" is composed of eight residues from histones H2B and H2A that are known to provide a docking interface for many proteins. For instance, PRC1, RCC1, and Orc1 bind to the nucleosome acidic patch through a common binding motif consisting of a loop region with an arginine residue that inserts directly into the acidic pocket (31-33). Though these regions are primarily unstructured and basic, this docking requires the specific side chain structure of an arginine, which cannot be substituted by lysine, resulting in these regions being referred to as "arginine anchors" (34). In the context of remodelers, ISWI uses an arginine anchor within its catalytic ATPase subunit to relieve an autoinhibitory mechanism following nucleosome binding (29). Members of the SWI/SNF family of enzymes also use an arginine anchor motif within their conserved Snf5-like subunits to anchor the complex to the octamer during remodeling activity (35). Recent cryoEM structures of INO80 family members have predicted key interactions with the nucleosomal acidic patch (36), and this domain has been found to be key for remodeling activity (29).

Here we investigate the role of the nucleosome acidic patch in SWR1C dimer exchange. Employing a quantitative FRET-based assay and a nucleosomal substrate harboring substitutions within acidic patch residues, we find that the acidic patch plays an essential role in SWR1C dimer exchange. Loss of the acidic patch does not impact the ability of SWR1C to bind to nucleosomes, and nucleosomes that lack the acidic patch retain the ability to stimulate SWR1C ATPase activity. We identify a putative arginine anchor domain within the Swc5 subunit of SWR1C, and we show that this domain is essential for dimer exchange in vitro and the function of SWR1C in vivo. The results indicate that SWR1C activity relies on communication between the Swc5 arginine anchor and the nucleosome acidic patch to functionally couple ATPase activity to enable nucleosome editing.

\section{Material and Methods}

\section{Yeast strains and culture conditions}


The yeast strain $S W R 1-3 x F L A G$ htz1 $\triangle$ (yEL190) was a gift from Ed Luk (SUNY Stony Brook). Deletion of SWC5 was constructed by standard gene replacement with a Hygromycin B cassette

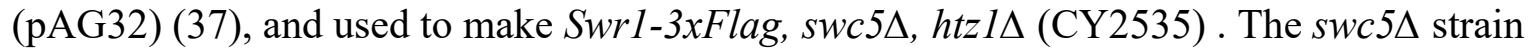
(Y03048) used for spot assays was purchased from Euroscarf.

Spot assays were performed with the $s w c 5 \Delta$ strain (Y03048) transformed with an empty CEN ARS URA3 vector PRS416, or ones containing WT and mutant SWC5. Cultures were diluted to an $\mathrm{OD}_{600}$ of 1 and then serially diluted 1:10. A dilution (7ul) was spotted onto synthetic complete media lacking uracil with and without $3 \%$ formamide. Plates were incubated for 3 days before imaging.

\section{Plasmid Construction}

The vector (pQE80L) was used for recombinant Swc5 expression. The SWC5 gene and SWC 59${ }^{303}$ were inserted in frame with the N-terminal 6x histidine tag through Gibson assembly (38) after digesting the vector with BamHI and SphI. Once WT and truncated Swc5 were in frame, the alanine mutants [swc5 (RRKR-4A) and $s w c 5(L D W-3 A)]$ were generated using QuickChange site directed mutagenesis. The pRS416 CEN ARS URA3 vector was digested with XbaI and SacI to allow for insertion of the SWC5 gene with 500bp upstream of the start site and $500 \mathrm{bp}$ downstream of the stop. This region was initially PCR amplified from a W303 strain where the primers had homology to the XbaI and SacI cut sites of the vector. Gibson assembly was used to ligate the vector and insert (CP1566) (38). Once the WT SWC5 gene was inserted, site directed mutagenesis was used on CP1566 to make swc5 RRKR-4A (CP1579) and swc5 LDW-3A (CP1580). swc5 79-303 construct was generated using CP1566 to PCR amplify the 500bp upstream stretch of DNA and start site of SWC5 and PCR amplifying another DNA fragment starting from Swc5 K79 to 500bp downstream of the stop. Primers were designed so each PCR fragment had homology to each other and the pRS416 vector at XbaI and SphI so that the two fragments and the pRS416 vector could be ligated together by Gibson assembly. Site directed mutagenesis was used to generate cysteine mutants used for Oregon Green labeling, as well as for single amino acid substitution used for assembly of acidic patch mutants (APM) octamers H2A-E63A, H2A-E65A, H2A-D74A, H2A-D92A, H2A-D93A, H2A-E94A, H2B-E109A, H2BE117A.

\section{Nucleosome reconstitution}

Histones and nucleosomes were generated as described previously $(39,40)$. Hybrid octamers were generated using Saccharomyces cerevisiae H2A and H2B with Xenopus larvus or human histone $\mathrm{H} 3$ and $\mathrm{H} 4$. WT and acidic patch mutant nucleosomes used for quenching and gel shifts contained yH2B-S115C or xH4-Q27C labeled with Oregon Green ${ }^{\mathrm{TM}} 488$ Malemide (ThermoFisher). Histones labelled with $\mathrm{Cy} 3$ or Oregon Green were generated as described previously (41). The Widom 601 nucleosome positioning sequence was used to generate either $77 \mathrm{N0}$ or 0N0 DNA templates (42). 77N0 templates were used in dimer exchange and ATPase assays while the $0 \mathrm{~N} 0$ template was used for nucleosome binding and quenching assays.

\section{SWR1C purification}

Swr1-3xFlag yeast strains were grown in $6 \times 2 \mathrm{~L}$ of YEPD, supplemented with adenine at $30^{\circ} \mathrm{C}$ until reaching an $\mathrm{OD}_{600}$ of 3-6. Cultures were centrifuged and pellets were noodled into liquid nitrogen and stored at $-80^{\circ} \mathrm{C}$. SWR1C was purified as previously described $(18,40)$ with minor 
modifications. A PM 100 cryomill was used to lyse the harvested yeast noodles with $6 \times 1 \mathrm{~min}$ cycles at $400 \mathrm{rpm}$ then stored at $-80^{\circ} \mathrm{C}$. Lysed cell powder $(\sim 200 \mathrm{~mL})$ were resuspended in 200mL 1x HC lysis buffer ([25 mM HEPES-KOH (pH 7.6), 10\% glycerol, 1 mM EDTA, 300 $\mathrm{mM} \mathrm{KCl}, 0.01 \%$ NP40, $10 \mathrm{mM} \beta$-glycerophosphate, $1 \mathrm{mM}$ Na-butyrate, $0.5 \mathrm{mM} \mathrm{NaF}, 1 \mathrm{mM}$ DTT plus $1 \times$ protease inhibitors [PI 1mM PMSF, $1 \mathrm{mM}$ benzamidine, $0.1 \mathrm{mg} / \mathrm{ml}$ pepstatin A, 0.1 $\mathrm{mg} / \mathrm{ml}$ leupeptin, $0.1 \mathrm{mg} / \mathrm{ml}$ chymostatin)] in a $1 \mathrm{~L}$ beaker with a stir bar at $4^{\circ} \mathrm{C}$. Thawed cells were centrifuged for 2 hours at $35000 \mathrm{rpm}$ in a Ti45 rotor at $4^{\circ} \mathrm{C}$ then whole cell extract was transferred to a $250 \mathrm{ml}$ falcon tube along with anti-Flag $\mathrm{M} 2$ agarose resin ( $1 \mathrm{~mL}$ bed volume) and nutated at $4^{\circ} \mathrm{C}$ for 3 hours. Whole cell extract and resin were centrifuged at $750 \mathrm{rcf}$ and supernatant was aspirated off. Resin was transferred to $25 \mathrm{ml}$ gravity column and washed with $4 \mathrm{x}$ 25 B-0.5 buffer (25 mM HEPES, $\mathrm{pH}=7.6,1 \mathrm{mM}$ EDTA, $2 \mathrm{mM} \mathrm{MgCl} 2,10 \mathrm{mM} \beta$ glycerophosphate, $1 \mathrm{mM}$ Na-butyrate, $0.5 \mathrm{mM} \mathrm{NaF}, 500 \mathrm{mM} \mathrm{KCl}, 10 \%$ glycerol, $0.01 \% \mathrm{NP} 40$ and $1 \mathrm{x}$ PI) then washed with 3x 5ml B-0.1 buffer (25 mM HEPES, $\mathrm{pH}=7.6,1 \mathrm{mM}$ EDTA, 2 $\mathrm{mM} \mathrm{MgCl} 2,10 \mathrm{mM} \beta$-glycerophosphate, $1 \mathrm{mM}$ Na-butyrate, $0.5 \mathrm{mM} \mathrm{NaF}, 100 \mathrm{mM} \mathrm{KCl}, 10 \%$ glycerol, $0.01 \% \mathrm{NP} 40$ and 1x PI). For purification of SWR $1 \mathrm{C}^{\mathrm{swc} 5 \Delta}$ where recombinant Swc5 is added back, rSwc 5 would be diluted to $5 \mathrm{uM}$ in $2 \mathrm{~mL}$ of B- 0.1 and added to resin for $15 \mathrm{~min}$ at $4^{\circ} \mathrm{C}$ followed by $2 \times 5 \mathrm{~mL}$ B- 0.5 washes then $2 \times 5 \mathrm{~mL}$ B- 0.1 washes. SWR $1 \mathrm{C}$ was eluted by nutating resin in $1 \mathrm{~mL}$ B- 0.1 with $0.5 \mathrm{mg} / \mathrm{ml} 3 \times$ Flag peptide for 1 hour. Elution is done twice and both are combined and concentrated using a $100 \mathrm{kDa}$ cutoff Amicon Ultra- $0.5 \mathrm{~mL}$ centrifugal filter (Millipore). After concentrating down to 100ul, 3x Flag peptide is removed by bringing volume back to 500 ul with B-0.1 three more times in the amicon. After the Flag peptide is removed, concentrated SWR1C was aliquoted, flash frozen, and stored at $-80^{\circ} \mathrm{C}$. SWR1C concentration was determined by SDS-PAGE using a BSA (NEB) standard titration followed by SYPRO Ruby (Thermo Fisher Scientific) staining and quantification using ImageQuant 1D gel analysis.

\section{Recombinant Swc5 Purification}

Swc5 and variants were first cloned into a pQE80L expression vector in frame with a six histidine N-Terminal tag through Gibson assembly. Plasmids were transformed into Rosetta 2 DE3 competent cells (Novagen). 1L cultures of 2x YT (Yeast extract, tryptone) were grown at $37^{\circ} \mathrm{C}$ to $\mathrm{OD}_{600}$ of $0.5-0.7$ before adding $0.4 \mathrm{mM}$ IPTG. After adding IPTG, cells continued to shake at room temperature for 3 hours. Cells were harvested by centrifugation at $4^{\circ} \mathrm{C}$ and $1 \mathrm{~L}$ of Swc5 bacterial pellet was resuspended in $20 \mathrm{~mL}$ lysis buffer $(10 \mathrm{mM}$ Imidazole, $50 \mathrm{mM}$ Tris $\mathrm{pH}$ 8.0, 300mM NaCl, 1mM PMSF, 1 mM DTT) before being flash frozen in liquid nitrogen and stored at $-80^{\circ} \mathrm{C}$. Pellets were thawed in a $37^{\circ} \mathrm{C}$ water bath then fresh $0.5 \mathrm{mM}$ PMSF and $1 \mathrm{mM}$ Benzamidine were added. Cells were sonicated 5 x 30 seconds at $15 \%$ power, incubating on ice for $1 \mathrm{~min}$ between sonication intervals. Lysate was then centrifuged at $14000 \mathrm{rpm}$ in a JA-17 rotor at $4^{\circ} \mathrm{C}$ for $25 \mathrm{~min}$. Whole cell extract was incubated with $800 \mathrm{ul}$ of HisPur Ni-NTA resin slurry, that had been prewashed with lysis buffer, for 2 hours at $4^{\circ} \mathrm{C}$ on nutator. The resin and whole cell extract mixture was centrifuged at 750 rcf for 5 minutes. The supernatant was aspirated and the resin was placed in a $25 \mathrm{ml}$ gravity column and washed with $5 \mathrm{x} 10 \mathrm{ml}$ wash buffer (500mM NaCl, 10mM Imidazole, 50mM Tris pH8.0). Swc5 was eluted by capping the gravity column and adding $400 \mathrm{ul}$ elution buffer $(500 \mathrm{mM} \mathrm{NaCl}, 300 \mathrm{mM}$ Imidazole, $50 \mathrm{mM}$ Tris $\mathrm{pH}$ 8.0) to resin and incubating at $4^{\circ} \mathrm{C}$ for $10 \mathrm{~min}$ then collecting the flow through. The Swc5 eluate was dialyzed against $2 \times 250 \mathrm{~mL}$ of storage buffer $(50 \mathrm{mM} \mathrm{NaCl}, 50 \mathrm{mM}$ Tris pH $7.4,10 \%$ glycerol) then flash frozen and stored at $-80^{\circ} \mathrm{C}$. Concentration was determined by BSA standard on a SDS PAGE gel. 


\section{Nucleosome dimer exchange assays}

FRET based dimer exchanges assays were performed as previously described (40) using an ISS PC1 spectrofluorometer or a Tecan Infinite M1000 PRO microplate reader. The nucleosome remodeling reactions $(50-100 \mathrm{ul})$ were performed in remodeling buffer $(25 \mathrm{mM}$ HEPES, $\mathrm{pH}=$ 7.6, $0.2 \mathrm{mM}$ EDTA, $5 \mathrm{mM} \mathrm{MgCl} 2,70 \mathrm{mM} \mathrm{KCl}, 1 \mathrm{mM}$ DTT) at room temperature with $30 \mathrm{nM}$ SWR1C, 10nM 77N0 Cy3 H2A-Cy5 Nucleosome, 70nM H2A.Z/H2B dimers, and 1mM ATP (to start reaction). Dimer exchange was observed by exciting the $\mathrm{Cy} 3$ fluorophore at $530 \mathrm{~nm}$ and monitoring the Cy5 emission 670.

Gel based dimer exchanged assays used a modified protocol from a previous study $(14,22)$. A 150ul dimer exchange reaction contained 30nM SWR1C, 10nM 77N0 nucleosomes (WT or APM), and 70nM H2A.Z flag/H2B in remodeling buffer with $0.1 \mathrm{mg} / \mathrm{ml} \mathrm{BSA}$. The reaction was performed at room temperature and $20 \mathrm{ul}$ of the reaction was taken at each time point (time 0 is before $1 \mathrm{mM}$ ATP is added) and quenched with 1ug of plasmid DNA to separate SWR1C and dimers from the nucleosome. Each time point was stored on ice until last sample was quenched. Each time point was loaded onto a $6 \% 0.5 \mathrm{x}$ TBE Native PAGE gel and electrophoresed for $90 \mathrm{~min}$ at $120 \mathrm{~V}$. Gels were stained with SYBR gold and imaged on a GE Typhoon.

\section{Swc5 binding assays}

Gel shifts with recombinant WT or Swc5 derivatives were performed in $15 \mathrm{ul}$ reactions. Reactions contained varying amounts of Swc5 in a total volume of $5 \mathrm{ul}$ of storage buffer $(10 \%$ glycerol, $50 \mathrm{mM}$ Tris $7.4,50 \mathrm{mM} \mathrm{NaCl}), 0.25 \mathrm{ul} 300 \mathrm{nM} 0 \mathrm{~N} 0$ nucleosomes, 6.15 water, $0.6 \mathrm{ul} 50 \%$ glycerol, and 3ul 5x binding buffer (125mM HEPES 7.3, 250mM NaCl, 25mM MgCl $2,0.05 \%$ Tween $20,0.5 \mathrm{mg} / \mathrm{ml} \mathrm{BSA,} \mathrm{5mM} \mathrm{DTT).} \mathrm{Reaction} \mathrm{incubated} \mathrm{in} \mathrm{the} \mathrm{dark} \mathrm{at} \mathrm{room} \mathrm{temp} \mathrm{for} 30 \mathrm{~min}$. Samples were loaded onto 6\% Native PAGE gels and electrophoresed at 230V for 1 hour. Gels were imaged on a GE typhoon imager and binding was calculated by measuring the disappearance of the free nucleosome band. GraphPad Prism 8 was used to generate binding curves.

Swc5 fluorescent quenching assays were assembled similar to the gel shift assays but in 40ul reactions with 10nM 0N0 Oregon Green nucleosomes (8ul 5x binding buffer, 1.3ul 300nM Nucleosomes, $11.7 \mathrm{ul}$ water, $19 \mathrm{ul} \mathrm{Swc5/storage} \mathrm{buffer).} \mathrm{Master} \mathrm{mixes} \mathrm{of} \mathrm{each} \mathrm{reaction} \mathrm{incubated}$ for 30min at room temp in the dark before being loaded into 384 well flat black plate (PerkinElmer) and scanning each well 20 times on a Tecan Spark plate reader at 488nm excitation and 530nm emission. Quenching was calculated by taking the average relative fluorescence units (RFU) of each Swc5 containing well and dividing RFU average of the nucleosome only wells. The normalized fluorescence was plotted using Prism 8.

\section{ATPase assay}

ATPase activity was measured using either a phosphate binding protein reporter assay or NADH coupled assay. Each assay showed identical fold stimulation by nucleosomes when ATPase stimulation was normalized to basal activity of WT SWR1C or SWR1C ${ }^{\text {swc5s }}$ complexes reconstituted with recombinant Swc5. For the phosphate sensor assay $(18,43)$, 20ul reactions were prepared with 1nM SWR1C mixed with 0.5uM phosphate sensor (fluorophore-conjugated phosphate binding protein, ThermoFisher) in reaction buffer [25 mM HEPES-KOH (pH 7.6), 0.5 
mM EGTA, $0.1 \mathrm{mM}$ EDTA, $4.3 \mathrm{mM} \mathrm{MgCl}$, $0.14 \mathrm{ug} / \mathrm{ul} \mathrm{BSA,} 64 \mathrm{mM} \mathrm{NaCl}, 10 \%$ glycerol, and $0.02 \%$ NP-40]. Reaction containing nucleosome and H2A.Z/H2B dimers had concentrations of $15 \mathrm{nM}$ and $30 \mathrm{nM}$ respectively. Reactions were initiated by adding 100uM ATP and monitored by the measuring the fluorescence of the MDCC fluorophore on a Tecan M1000 infinite by exciting at $405 \mathrm{~nm}$ and setting the emission filter to $460 \mathrm{~nm}$. ATP was pre-treated with a "Pi Mop" to remove free phosphate as previously described $(18,43)$. Time points were collected every 4 seconds over a ten minute time course. Rates were obtained by taking the slope of the linear region (100-500 seconds) of three replicates assays for each condition.

The NADH coupled ATPase was performed using a modified version of a published protocol (44). This assay measures ATP hydrolysis by monitoring loss of NADH fluorescence, which is reduced as ADP formation converts $\mathrm{NADH}$ to $\mathrm{NAD}^{+}$(45). 20nM SWR1C, 75nM nucleosomes, and $150 \mathrm{nM} \mathrm{H} 2 \mathrm{~A} . \mathrm{Z} / \mathrm{H} 2 \mathrm{~B}$ dimers were mixed in $50 \mathrm{ul}$ reactions with a buffer containing $20 \mathrm{U} / \mathrm{mL}$ pyruvate kinase (Sigma), (Sigma) 20U/mL lactate dehydrogenase, $0.67 \mathrm{mM}$ phosphoenolpyruvate (PEP), 0.1mM NADH, 50mM Tris 7.4, 0.5mM TCEP, $5 \mathrm{mM} \mathrm{MgCl}_{2}$, $100 \mathrm{mM} \mathrm{KCl}$. Reactions were loaded into a 384 well flat black plate (PerkinElmer) and started by adding 500uM ATP. Fluorescence was measured on a CLARIOstar microplate reader using an excitation of $339 \mathrm{~nm}$ and emission of 466 . Wells were measured every 4 seconds over a 10 minute time course, and ATPase rates were determined by taking the slope of the linear region of the trace (200-500seconds) and taking the average of two replicates. Significance was determined using a one-way ANOVA in GraphPad Prism 8.

\section{Results \\ SWR1C dimer exchange requires the nucleosomal acidic patch}

To investigate the role of the nucleosomal acidic patch on SWR1C dimer exchange activity, a fluorescence-based assay was initially employed. Recombinant nucleosomes were assembled with either wildtype (WT) histone octamers or with octamers that contained 8 alanine substitutions within the nucleosomal acidic patch (APM). The DNA template for nucleosome reconstitutions was a 224-bp fragment containing a "601" nucleosome positioning sequence (42) and an asymmetric, $77 \mathrm{bp}$ linker DNA to mimic the structure of a promoter-proximal nucleosome located next to a nucleosome depleted region (referred to as a 77N0 nucleosome; Figure 1A). These substrates contain a $\mathrm{Cy} 3$ fluorophore conjugated to the linker-distal end of the nucleosomal DNA, and a Cy5 fluorophore attached to an engineered cysteine residue within the histone $\mathrm{H} 2 \mathrm{~A} \mathrm{C}$-terminal domain. The $\mathrm{Cy} 3$ and $\mathrm{Cy} 5$ fluorophores are within an appropriate distance to function as a FRET pair, such that excitation of the Cy3 donor leads to efficient energy transfer to the Cy5 acceptor, as evidenced by the fluorescence emission peak at $670 \mathrm{~nm}$. The dimer exchange activity of SWR1C is monitored by following the decrease in the 670nm FRET signal due to eviction of the Cy5-labelled H2A/H2B dimer (see Figure 1A).

SWR1C dimer exchange reactions were performed under single turnover conditions (excess enzyme to nucleosomal substrate) and contained free H2A.Z/H2B dimers which act as an essential co-substrate. Addition of SWR1C to WT nucleosomes led to a rapid drop in FRET, showing the biphasic kinetics consistent with the sequential exchange of each of the two nucleosomal H2A/H2B dimers (Figure 1A, red curve) (40). As expected, reactions with SWR1C complex that lacked the essential Swc5 subunit, showed almost no change in the FRET signal (Figure 1A). Strikingly, incubation of SWR1C with the APM nucleosome also yielded 
little decrease in the FRET signal, indicating that an intact nucleosomal acidic patch is essential for SWR1C to catalyze H2A/H2B eviction (Figure 1A).

Since the FRET assay is limited to monitoring the eviction of nucleosomal $\mathrm{H} 2 \mathrm{~A} / \mathrm{H} 2 \mathrm{~B}$ dimers, we also employed a gel-based assay that monitors deposition of H2A.Z. In this assay, an unlabeled, 77N0 nucleosome was incubated with SWR1C, ATP, and free H2A.Z/H2B dimers in which the H2A.Z contains a 3xFLAG tag at its C-terminus. Reaction products are separated on native PAGE, and formation of the heterotypic (AZ) and homotopic (ZZ) nucleosomal products are detected by their reduced gel migration due to the 3xFLAG tag on H2A.Z. Similar to the FRET-based assay, the SWR1C-catalyzed deposition of H2A.Z was largely complete by 30-60 minutes for the WT nucleosomal substrate, but addition of SWR1C to the APM nucleosome led to negligible H2A.Z deposition after 60 minutes (Figure 1B). Together, these assays indicate that SWR1C requires an intact nucleosomal acidic patch for H2A.Z deposition.

The ATPase activity of SWR1C is stimulated by both its nucleosomal substrate and free H2A.Z/H2B dimers (18). Notably, the ATPase activity of SWR1C is not stimulated by an H2A.Z nucleosome, implying that SWR1C can distinguish product from substrate nucleosomes (18). To investigate whether the nucleosomal acidic patch plays a role in substrate recognition, SWR1C ATPase activity was examined in the presence of either WT or APM nucleosomes (Figure 1C). Consistent with previous work (18), SWR1C ATPase activity was stimulated nearly $2 \mathrm{x}$ by an $\mathrm{H} 2 \mathrm{~A}$-containing nucleosome, and addition of free $\mathrm{H} 2 \mathrm{~A} . \mathrm{Z} / \mathrm{H} 2 \mathrm{~B}$ dimers led to an additional hyperactivation (Figure 1C). SWR1C ATPase activity was also activated by the APM nucleosome, but the stimulation with reduced, and addition of the free H2A.Z/H2B dimers did not lead to a significant increase in stimulation (Figure 1C). Importantly, the decreased stimulation of ATPase activity was not due to a reduced nucleosomal binding affinity, as SWR1C binds with equal apparent affinity to both WT and APM nucleosomes in a gel-based binding assay (Figure 1D). Together these data suggest that the nucleosomal acidic patch plays a role in both stimulating ATPase activity, as well as coupling ATPase hydrolysis with histone dimer exchange activity.

\section{Conserved arginine patch on Swc5 is essential for dimer exchange activity}

Recently, a potential arginine anchor domain was identified within the N-terminal, acidic domain of the SWR1C subunit, Swc2. However, removal of this domain (Swc2-ZN) had only a modest impact on the dimer exchange activity of SWR1C, suggesting that additional subunits must contribute to recognition of the nucleosomal acidic patch (46). The Swc5 subunit is one candidate, as loss of Swc5 eliminates H2A.Z deposition (19). Previous studies of Swc5 identified an acidic $\mathrm{N}$-terminal domain that interacts preferentially with $\mathrm{H} 2 \mathrm{~A} / \mathrm{H} 2 \mathrm{~B}$ dimers and a conserved C-terminal domain, termed BCNT, that is essential for SWR1C dimer exchange activity $(27,47)$. An alignment of Swc5 homologs revealed a region adjacent to the BCNT domain, containing a conserved basic region that has the hallmarks of an arginine anchor (Figure 2A).

To investigate the role of this Swc5 arginine-rich domain, alanine substitutions were created in four basic residues (RRKR), and this derivative was recombinantly expressed and reconstituted into a SWR1C complex purified from a $s w c 5$ deletion strain (SWR1C ${ }^{\mathrm{swc} 5 \Delta}$ ). In addition, SWR1C was reconstituted with wildtype Swc5, a Swc5 derivative lacking the acidic N- 
terminal domain (Swc5 ${ }^{79-303}$ ), and a derivative harboring alanine substitutions in the essential BCNT domain (Swc5 $\left.5^{\mathrm{LDW}-3 \mathrm{~A}}\right)$. In all cases, derivatives were incorporated into SWR1C complexes with equal efficiencies (Supplementary Figure S1). Consistent with previous studies, reconstitution with wildtype Swc5 fully restored dimer exchange activity to SWR $1 C^{\mathrm{swc} 5 \Delta}$ (Figure 2B). In contrast, SWR1C harboring Swc $5^{\text {LDW-3A }}$ had no detectable exchange activity, and the complex that lacks the N-terminal, acidic domain of Swc5 (Swc5 $\left.{ }^{79-303}\right)$ exhibited a modest defect, as expected from previous studies (Figure 2B) (27). Strikingly, SWR1C that contained the Swc5 ${ }^{\text {RRKR-4A }}$ derivative showed minimal activity in the FRET-based exchange assay (Figure 2B), and this complex exhibited only $\sim 10 \%$ the activity of wildtype SWR1C in the gel-based H2A.Z deposition assay (Supplementary Figure S2). Dimer exchange activity for each SWR1C complex was also mirrored by their ATPase activity (Figure 2C). For instance, the ATPase activity of the Swc $5^{\text {LDW-3A }}$ complex was not stimulated by either nucleosomes or free H2A.Z/H2B dimers, and the complex harboring Swc $5^{79-303}$ had wildtype levels of ATPase activity (Figure 2C). Disruption of the Swc5 arginine-rich domain nearly eliminated the nucleosome-stimulated ATPase activity of SWR1C (Figure 2C; Swc5 ${ }^{\text {RRKR-4A }}$ ). Together these data indicate that the arginine-rich domain of Swc5 is key for both ATPase activity and H2A.Z deposition.

\section{Swc5 interacts with the nucleosomal acidic patch}

To further investigate whether the arginine-rich domain within Swc5 binds to the nucleosomal acidic patch, in vitro binding assays were performed with nucleosome core particles (0N0) and recombinant Swc5 (Figure 3). Increasing amounts of Swc5 were incubated with nucleosomes, and binding was visualized by native PAGE. Addition of wildtype Swc5 to nucleosomes led to formation of discrete complexes with an apparent Kd of 178nM (Figure 3A). Likewise, binding of Swc $5^{\mathrm{LDW}-3 \mathrm{~A}}$, which has a disrupted BCNT domain, bound to nucleosomes with nearly identical affinity, compared to wildtype Swc5 (Figure 3B). Importantly, high affinity binding of Swc5 to nucleosomes required an intact acidic patch, with binding reduced to $\sim 800 \mathrm{nM}$ when an APM nucleosome was used as the substrate (Figure 3C). Furthermore, the Swc5 ${ }^{\text {RRKR-4A }}$ derivative had little nucleosome binding activity, with an apparent Kd $\sim 1900 \mathrm{nM}$ (Figure 3D). Together these data demonstrate that Swc5 has nucleosome binding activity, and the data are consistent with direct interactions between the Swc5 arginine-rich domain and the nucleosomal acidic patch.

While the gel shift assays establish that Swc5 can bind to nucleosomes, data from these assays can only indirectly link the nucleosomal acidic patch to the Swc5 arginine-rich domain. In order to probe for more direct interactions, a fluorescence quenching assay was employed (Figure 4A). These assays exploit nucleosomes harboring a site-specific, Oregon Green fluorophore whose emission is sensitive to the chemical environment whereby fluorescence is quenched by protein binding $(48,49)$. Nucleosome core particles were reconstituted that contained an Oregon Green fluorophore covalently attached to either the histone H4 N-terminal domain (H4-tail) or to a residue directly adjacent to the nucleosomal acidic patch (Figure 4A). Titration of wildtype Swc5 led to the concentration-dependent quenching of the Oregon Green fluorophore positioned at the acidic patch, but no quenching was observed for the fluorophore located on the H4-tail (Figure 4B). Importantly, Swc5 did not quench the acidic patch probe when this substrate also harbored alanine substitutions within the acidic patch (Figure 4B; APM nucleosome). The Swc5 $5^{\text {LDW-3A }}$ derivative also showed specific quenching of the acidic patch 
probe, but the Swc $5^{\text {RRKR-4A }}$ derivative was clearly defective for specific binding in this quenching assay (Figure 4C,D). Together these results are consistent with a direct interaction between the arginine-rich domain of Swc5 and the nucleosomal acidic patch, an interaction that is essential for SWR1C dimer exchange activity.

\section{The Swc5 arginine-rich domain is required for SWR1C function in vivo}

Previous studies have shown that yeast lacking functional SWR1C are inviable on media containing $3 \%$ formamide $(13,27)$. To investigate the functional role of the Swc5 arginine-rich domain in vivo, growth assays were performed with isogenic swc $5 \Delta$ strains that harbor low copy vectors that express different Swc5 derivatives (Figure 5). As expected, the $s w c 5 \Delta$ strain grew well on rich media, but was inviable on formamide media (vector), while cells expressing wildtype Swc5 grew well on both media (Swc5). Consistent with a previous study, the derivative with a disrupted BCNT domain was inviable on formamide media (Swc5 ${ }^{\mathrm{LDW}-3 \mathrm{~A}}$ ), and the Swc $5^{79-303}$ derivative that lacks the acidic N-terminal domain showed a moderate growth defect (27). Importantly, the Swc5 $5^{\text {RRKR-4A }}$ derivative had a severe growth defect on formamide media, consistent with an essential role of the arginine rich region in SWR1C function. Notably, survival on formamide media appears to be an excellent measurement of the in vitro dimer exchange activity of SWR1C, where the growth defects of each derivative is directly proportional to their in vitro activity.

\section{DISCUSSION}

The nucleosomal acidic patch has emerged as a key binding pocket for nearly all ATPdependent chromatin remodeling complexes. For some enzymes, like ISWI, interactions between the ATPase subunit and the acidic patch are required for optimal ATPase and nucleosome sliding activities, but they also help to relieve an inhibitory mechanism that also involves the histone H4 N-terminal domain $(29,30,50)$. The nucleosomal acidic patch is also essential for remodeling by SWI/SNF family members, and in this case the two acidic patches on the nucleosome are contacted by two different subunits -Snf5 (human SMARCAD1) and the Snf2 (human BRG1) ATPase $(35,51)$. Since a Snf2-like ATPase is sufficient for many of the remodeling activities of SWI/SNF family members $(52,53)$, it seems likely that the anchoring of Snf2 to the acidic patch is key for remodeling activity, while the Snf5-acidic patch interaction plays a less well-characterized, accessory role. Here, we have found that the ATP-dependent deposition of H2A.Z by the SWR1C enzyme also requires an intact acidic patch. In this case, the acidic patch does not appear to regulate the binding of SWR1C to nucleosomes, and nucleosomes that lack an intact acidic patch retain much of the ability to stimulate the SWR1C ATPase activity. Thus, the role of the acidic patch for SWR1C activity appears similar to that of SWI/SNF, where anchoring to the acidic patch facilitates the coupling of ATP hydrolysis to the remodeling mechanism, such as DNA translocation in the case of SWI/SNF, or $\mathrm{H} 2 \mathrm{~A} / \mathrm{H} 2 \mathrm{~B}$ dimer eviction for SWR1C.

A recent cryoEM structure of a SWR1C-nucleosome complex suggests that the enzyme has two major nucleosomal contacts - the Swr1 ATPase lobes interact with nucleosomal DNA $\sim 2$ helical turns from the nucleosomal dyad (SHL2.0), and the Swc6/Arp6 module interacts with DNA at the nucleosomal edge (20). These contacts encompass the DNA gyre that wraps the $\mathrm{H} 2 \mathrm{~A} / \mathrm{H} 2 \mathrm{~B}$ dimer that is destined to be exchanged in the first round of catalysis (the linker distal 
dimer (40)). Each of the two nucleosomal acidic patches also appear to make contacts with SWR1C subunits. An arginine residue from Arp6 appears to contact the acidic patch on the targeted, linker distal dimer, while Swc2 extends across the two DNA gyres to interact with the acidic patch on the distal, acidic patch (20). While functional analyses of the Arp6 interaction have not yet been reported, a recent study found that disruption of the putative Swc2 arginine anchor led to only a minor defect in SWR1C exchange activity (46). Here, we identified an arginine-rich domain within the Swc5 subunit that appears to directly interact with the nucleosomal acidic patch and is essential for SWR1C activity. Unfortunately, the Swc5 subunit was not visualized in the cryoEM structure (20). Disruption of this putative arginine anchor disrupts the nucleosome binding activity of Swc5, cripples the ATPase activity of SWR1C, and nearly eliminates the ability of SWR1C to evict nucleosomal H2A/H2B and to deposit H2A.Z. These data suggest a compelling model in which this arginine-rich domain of Swc5 makes a key anchoring contact with the $\mathrm{H} 2 \mathrm{~A} / \mathrm{H} 2 \mathrm{~B}$ dimer that is targeted for eviction, helping to activate the Swr1 ATPase and couple ATPase activity to dimer eviction.

Previous functional studies of Swc5 identified two distinct functional domains. The conserved, C-terminal BCNT domain is essential for Swc5 function, as amino acid substitutions within this domain eliminate SWR1C ATPase and H2A.Z deposition activity (27). Notably, an intact BCNT domain is not required for assembly of Swc5 into SWR1C. How BCNT functions is currently unknown, but it seems likely that it interacts directly with the Swr1 ATPase, activating the enzyme when it has engaged the H2A nucleosomal substrate. Indeed, a previous crosslinking study found that the BCNT region contacts Swr1, adjacent to the second ATPase lobe (54). Secondly, an Nterminal acidic region (1-79) of Swc5 contains a histone chaperone domain that binds to free $\mathrm{H} 2 \mathrm{~A} / \mathrm{H} 2 \mathrm{~B}$ dimers, and this domain has been proposed to facilitate removal of the nucleosomal $\mathrm{H} 2 \mathrm{~A} / \mathrm{H} 2 \mathrm{~B}$ dimer (47). Consistent with this model, we and others find that deletion of this N-terminal domain leads to a modest defect in H2A.Z deposition, but this domain is not important for ATPase activity. Notably, we find that neither the acidic N-terminus or the BCNT domain impact the nucleosome binding activity of Swc5, nor are they required for interaction with the nucleosomal acidic patch, as assayed by fluorescence quenching. Thus, the arginine-rich region identified in this work functions as a novel, essential domain within the Swc5 subunit.

A previous study did not detect nucleosome binding by recombinant Swc5 (27). We did find that different preparations of recombinant Swc5 showed large differences in their apparent affinities for nucleosomes, with some preps showing low micromolar affinities. Variabilities among Swc5 preparations appear to be due to the highly acidic N-terminal domain, as the Swc $5^{79-303}$ derivative shows reproducible, high affinity binding activity (Supplementary Figure S3A). Nucleosomal binding of Swc5 ${ }^{79-303}$, like full-length Swc5, requires an intact nucleosomal acidic patch and the arginine-rich domain (Supplementary Figure S3B,C). Furthermore, the Swc5 ${ }^{79-303}$ derivative was active in the fluorescence quenching assay for nucleosomal acidic patch interaction (Supplementary Figure S4).

The mammalian homolog of Swc5 is Craniofacial development protein 1 (Cfdp1), although there is currently a lack of information on the role of Cfdp 1 in development. Interestingly, a survey of Cfdp1 mutations identified in the TCGA transCancer atlas reveals a large number of missense substitutions within the BCNT domain, as well as a cluster of substitutions directly adjacent to the arginine-rich domain. The majority of these Cfdp1 
alterations are associated with uterine endometrial carcinomas, suggesting that Cfdp1, and likely the SRCAP remodeler, are key for suppressing cancer initiation or progression in this cell type.

\section{ACKNOWLEDGMENTS}

We thank Ed Luk (SUNY Stony Brook) for the kind gift of the Swr1-3xFLAG strain; Jessica Feldman for vector pQE80L, and members of the Peterson lab for helpful discussions.

\section{FUNDING}

This work was supported by the National Institutes of Health [R35-GM122519 to C.L.P.]

Conflict of Interest Statement - None declared

\section{REFERENCES}

1. Luger,K., Mäder,A.W., Richmond,R.K., Sargent,D.F. and Richmond,T.J. (1997) Crystal structure of the nucleosome core particle at $2.8 \AA$ resolution. Nature, 389, 251-260.

2. Clapier,C.R., Iwasa,J., Cairns,B.R. and Peterson,C.L. (2017) Mechanisms of action and regulation of ATP-dependent chromatin-remodelling complexes. Nat Rev Mol Cell Biol, 18, 407-422.

3. Albert,I., Mavrich,T.N., Tomsho,L.P., Qi,J., Zanton,S.J., Schuster,S.C. and Pugh,B.F. (2007) Translational and rotational settings of H2A.Z nucleosomes across the Saccharomyces cerevisiae genome. Nature, 446, 572-576.

4. Barski,A., Cuddapah,S., Cui,K., Roh,T.-Y., Schones,D.E., Wang,Z., Wei,G., Chepelev,I. and Zhao,K. (2007) High-Resolution Profiling of Histone Methylations in the Human Genome. Cell, 129, 823-837.

5. Hartley,P.D. and Madhani,H.D. (2009) Mechanisms that Specify Promoter Nucleosome Location and Identity. Cell, 137, 445-458.

6. Boyarchuk,E., Montes de Oca,R. and Almouzni,G. (2011) Cell cycle dynamics of histone variants at the centromere, a model for chromosomal landmarks. Current Opinion in Cell Biology, 23, 266-276.

7. Xu,Y., Ayrapetov,M.K., Xu,C., Gursoy-Yuzugullu,O., Hu,Y. and Price,B.D. (2012) Histone H2A.Z Controls a Critical Chromatin Remodeling Step Required for DNA DoubleStrand Break Repair. Molecular Cell, 48, 723-733.

8. Meneghini,M.D., Wu,M. and Madhani,H.D. (2003) Conserved Histone Variant H2A.Z Protects Euchromatin from the Ectopic Spread of Silent Heterochromatin. Cell, 112, 725736.

9. Guillemette,B., Bataille,A.R., Gévry,N., Adam,M., Blanchette,M., Robert,F. and Gaudreau,L. (2005) Variant Histone H2A.Z Is Globally Localized to the Promoters of Inactive Yeast Genes and Regulates Nucleosome Positioning. PLoS Biol, 3, e384. 
10. Dion,M.F., Kaplan,T., Kim,M., Buratowski,S., Friedman,N. and Rando,O.J. (2007)

Dynamics of Replication-Independent Histone Turnover in Budding Yeast. Science, 315, 1405-1408.

11. Raisner,R.M., Hartley,P.D., Meneghini,M.D., Bao,M.Z., Liu,C.L., Schreiber,S.L., Rando,O.J. and Madhani,H.D. (2005) Histone Variant H2A.Z Marks the 5' Ends of Both Active and Inactive Genes in Euchromatin. Cell, 123, 233-248.

12. Creyghton,M.P., Markoulaki,S., Levine,S.S., Hanna,J., Lodato,M.A., Sha,K., Young,R.A., Jaenisch,R. and Boyer,L.A. (2008) H2AZ Is Enriched at Polycomb Complex Target Genes in ES Cells and Is Necessary for Lineage Commitment. Cell, 135, 649-661.

13. Kobor,M.S., Venkatasubrahmanyam,S., Meneghini,M.D., Gin,J.W., Jennings,J.L., Link,A.J., Madhani,H.D. and Rine,J. (2004) A Protein Complex Containing the Conserved Swi2/Snf2-Related ATPase Swr1p Deposits Histone Variant H2A.Z into Euchromatin. PLOS Biology, 2, e131.

14. Mizuguchi,G., Shen,X., Landry,J., Wu,W.-H., Sen,S. and Wu,C. (2004) ATP-Driven Exchange of Histone H2AZ Variant Catalyzed by SWR1 Chromatin Remodeling Complex. Science, 303, 343-348.

15. Johnston,H., Kneer,J., Chackalaparampil,I., Yaciuk,P. and Chrivia,J. (1999) Identification of a Novel SNF2/SWI2 Protein Family Member, SRCAP, Which Interacts with CREBbinding Protein*. Journal of Biological Chemistry, 274, 16370-16376.

16. Fuchs,M., Gerber,J., Drapkin,R., Sif,S., Ikura,T., Ogryzko,V., Lane,W.S., Nakatani,Y. and Livingston,D.M. (2001) The p400 Complex Is an Essential E1A Transformation Target. Cell, 106, 297-307.

17. Ruhl,D.D., Jin,J., Cai,Y., Swanson,S., Florens,L., Washburn,M.P., Conaway,R.C., Conaway,J.W. and Chrivia,J.C. (2006) Purification of a Human SRCAP Complex That Remodels Chromatin by Incorporating the Histone Variant H2A.Z into Nucleosomes. Biochemistry, 45, 5671-5677.

18. Luk,E., Ranjan,A., FitzGerald,P.C., Mizuguchi,G., Huang,Y., Wei,D. and Wu,C. (2010) Stepwise Histone Replacement by SWR1 Requires Dual Activation with Histone H2A.Z and Canonical Nucleosome. Cell, 143, 725-736.

19. Wu,W.-H., Alami,S., Luk,E., Wu,C.-H., Sen,S., Mizuguchi,G., Wei,D. and Wu,C. (2005) Swc2 is a widely conserved H2AZ-binding module essential for ATP-dependent histone exchange. Nat Struct Mol Biol, 12, 1064-1071.

20. Willhoft,O., Ghoneim,M., Lin,C.-L., Chua,E.Y.D., Wilkinson,M., Chaban,Y., Ayala,R., McCormack,E.A., Ocloo,L., Rueda,D.S., et al. (2018) Structure and dynamics of the yeast SWR1-nucleosome complex. Science, 362, eaat7716. 
21. Watanabe,S., Tan,D., Lakshminarasimhan,M., Washburn,M.P., Erica Hong,E.-J., Walz,T. and Peterson,C.L. (2015) Structural analyses of the chromatin remodelling enzymes INO80-C and SWR-C. Nat Commun, 6, 7108.

22. Ranjan,A., Mizuguchi,G., FitzGerald,P.C., Wei,D., Wang,F., Huang,Y., Luk,E., Woodcock,C.L. and Wu,C. (2013) Nucleosome-free region dominates histone acetylation in targeting SWR1 to promoters for H2A.Z replacement. Cell, 154, 1232-1245.

23. Liang,X., Shan,S., Pan,L., Zhao,J., Ranjan,A., Wang,F., Zhang,Z., Huang,Y., Feng,H., Wei,D., et al. (2016) Structural basis of H2A.Z recognition by SRCAP chromatinremodeling subunit YL1. Nat Struct Mol Biol, 23, 317-323.

24. Lu,P.Y.T., Lévesque,N. and Kobor,M.S. (2009) NuA4 and SWR1-C: two chromatinmodifying complexes with overlapping functions and componentsThis paper is one of a selection of papers published in this Special Issue, entitled 30th Annual International Asilomar Chromatin and Chromosomes Conference, and has undergone the Journal's usual peer review process. Biochem. Cell Biol., 87, 799-815.

25. Gómez-Zambrano,Á., Crevillén,P., Franco-Zorrilla,J.M., López,J.A., Moreno-Romero,J., Roszak,P., Santos-González,J., Jurado,S., Vázquez,J., Köhler,C., et al. (2018) Arabidopsis SWC4 Binds DNA and Recruits the SWR1 Complex to Modulate Histone H2A.Z Deposition at Key Regulatory Genes. Molecular Plant, 11, 815-832.

26. Messina,G., Atterrato,M.T., Prozzillo,Y., Piacentini,L., Losada,A. and Dimitri,P. (2017) The human Cranio Facial Development Protein 1 (Cfdp1) gene encodes a protein required for the maintenance of higher-order chromatin organization. Sci Rep, 7, 45022.

27. Sun,L. and Luk,E. (2017) Dual function of Swc5 in SWR remodeling ATPase activation and histone H2A eviction. Nucleic Acids Res, 45, 9931-9946.

28. Dann,G.P., Liszczak,G.P., Bagert,J.D., Müller,M.M., Nguyen,U.T.T., Wojcik,F., Brown,Z.Z., Bos,J., Panchenko,T., Pihl,R., et al. (2017) ISWI chromatin remodellers sense nucleosome modifications to determine substrate preference. Nature, 548, 607611.

29. Gamarra,N., Johnson,S.L., Trnka,M.J., Burlingame,A.L. and Narlikar,G.J. (2018) The nucleosomal acidic patch relieves auto-inhibition by the ISWI remodeler SNF2h. eLife, 7, e35322.

30. Dao,H.T., Dul,B.E., Dann,G.P., Liszczak,G.P. and Muir,T.W. (2020) A basic motif anchoring ISWI to nucleosome acidic patch regulates nucleosome spacing. Nat Chem Biol, 16, 134-142.

31. Makde,R.D., England,J.R., Yennawar,H.P. and Tan,S. (2010) Structure of RCC1 chromatin factor bound to the nucleosome core particle. Nature, 467, 562-566.

32. McGinty,R.K., Henrici,R.C. and Tan,S. (2014) Crystal structure of the PRC1 ubiquitylation module bound to the nucleosome. Nature, 514, 591-596. 
33. De Ioannes,P., Leon,V.A., Kuang,Z., Wang,M., Boeke,J.D., Hochwagen,A. and Armache,K.J. (2019) Structure and function of the Orc1 BAH-nucleosome complex. Nat Commun, $10,2894$.

34. McGinty,R.K. and Tan,S. (2015) Nucleosome Structure and Function. Chem Rev, 115, 22552273.

35. Han,Y., Reyes,A.A., Malik,S. and He,Y. (2020) Cryo-EM structure of SWI/SNF complex bound to a nucleosome. Nature, 579, 452-455.

36. Eustermann,S., Schall,K., Kostrewa,D., Lakomek,K., Strauss,M., Moldt,M. and Hopfner,K.P. (2018) Structural basis for nucleosome remodeling by the INO80 complex. Nature, 556, 386-390.

37. Goldstein,A.L. and McCusker,J.H. (1999) Three new dominant drug resistance cassettes for gene disruption in Saccharomyces cerevisiae. Yeast, 15, 1541-1553.

38. Gibson,D.G., Young,L., Chuang,R.-Y., Venter,J.C., Hutchison,C.A. and Smith,H.O. (2009) Enzymatic assembly of DNA molecules up to several hundred kilobases. Nat Methods, 6 , $343-345$.

39. Luger,K., Rechsteiner,T.J. and Richmond,T.J. (1999) Expression and Purification of Recombinant Histones and Nucleosome Reconstitution. In Becker,P.B. (ed), Chromatin Protocols, Methods in Molecular Biology ${ }^{\mathrm{TM}}$. Humana Press, Totowa, NJ, pp. 1-16.

40. Singh,R.K., Fan,J., Gioacchini,N., Watanabe,S., Bilsel,O. and Peterson,C.L. (2019) Transient Kinetic Analysis of SWR1C-Catalyzed H2A.Z Deposition Unravels the Impact of Nucleosome Dynamics and the Asymmetry of Histone Exchange. Cell Rep, 27, 374386.e4.

41. Zhou,C.Y. and Narlikar,G.J. (2016) Chapter Five - Analysis of Nucleosome Sliding by ATPDependent Chromatin Remodeling Enzymes. In Marmorstein,R. (ed), Methods in Enzymology, Enzymes of Epigenetics, Part A. Academic Press, Vol. 573, pp. 119-135.

42. Lowary,P.T. and Widom,J. (1998) New DNA sequence rules for high affinity binding to histone octamer and sequence-directed nucleosome positioning1. Journal of Molecular Biology, 276, 19-42.

43. Brune,M., Hunter,J.L., Corrie,J.E.T. and Webb,M.R. (1994) Direct, Real-Time Measurement of Rapid Inorganic Phosphate Release Using a Novel Fluorescent Probe and Its Application to Actomyosin Subfragment 1 ATPase. Biochemistry, 33, 8262-8271.

44. Lin,C.-L., Chaban,Y., Rees,D.M., McCormack,E.A., Ocloo,L. and Wigley,D.B. (2017) Functional characterization and architecture of recombinant yeast SWR1 histone exchange complex. Nucleic Acids Research, 45, 7249-7260. 
45. Nørby,J.G. (1988) [11] Coupled assay of Na+,K+-ATPase activity. In Methods in Enzymology, Biomembranes Part P: ATP-Driven Pumps and Related Transport: The Na,K-Pump. Academic Press, Vol. 156, pp. 116-119.

46. Dai,L., Xiao,X., Pan,L., Shi,L., Xu,N., Zhang,Z., Feng,X., Ma,L., Dou,S., Wang,P., et al. (2021) Recognition of the inherently unstable H2A nucleosome by Swc2 is a major determinant for unidirectional H2A.Z exchange. Cell Reports, 35, 109183.

47. Huang,Y., Sun,L., Pierrakeas,L., Dai,L., Pan,L., Luk,E. and Zhou,Z. (2020) Role of a DEF/Y motif in histone $\mathrm{H} 2 \mathrm{~A}-\mathrm{H} 2 \mathrm{~B}$ recognition and nucleosome editing. PNAS, 117, 3543-3550.

48. Winkler,D.D., Luger,K. and Hieb,A.R. (2012) Chapter Eleven - Quantifying ChromatinAssociated Interactions: The HI-FI System. In Wu,C., Allis,C.D. (eds), Methods in Enzymology, Nucleosomes, Histones \& Chromatin Part A. Academic Press, Vol. 512, pp. 243-274.

49. Girish,T.S., McGinty,R.K. and Tan,S. (2016) Multivalent Interactions by the Set8 Histone Methyltransferase With Its Nucleosome Substrate. Journal of Molecular Biology, 428, 1531-1543.

50. Levendosky,R.F. and Bowman,G.D. (2019) Asymmetry between the two acidic patches dictates the direction of nucleosome sliding by the ISWI chromatin remodeler. eLife, $\mathbf{8}$, e45472.

51. Mashtalir,N., Suzuki,H., Farrell,D.P., Sankar,A., Luo,J., Filipovski,M., D’Avino,A.R., St. Pierre,R., Valencia,A.M., Onikubo,T., et al. (2020) A Structural Model of the Endogenous Human BAF Complex Informs Disease Mechanisms. Cell, 183, 802817.e24.

52. Saha,A., Wittmeyer,J. and Cairns,B.R. (2002) Chromatin remodeling by RSC involves ATPdependent DNA translocation. Genes Dev., 16, 2120-2134.

53. Yang,X., Zaurin,R., Beato,M. and Peterson,C.L. (2007) Swi3p controls SWI/SNF assembly and ATP-dependent H2A-H2B displacement. Nat Struct Mol Biol, 14, 540-547.

54. Nguyen,V.Q., Ranjan,A., Stengel,F., Wei,D., Aebersold,R., Wu,C. and Leschziner,A.E. (2013) Molecular Architecture of the ATP-Dependent Chromatin-Remodeling Complex SWR1. Cell, 154, 1220-1231.

55. Celauro,E., Carra,S., Rodriguez,A., Cotelli,F. and Dimitri,P. (2017) Functional analysis of the cfdp1 gene in zebrafish provides evidence for its crucial role in craniofacial development and osteogenesis. Experimental Cell Research, 361, 236-245.

56. Messina,G., Atterrato,M.T. and Dimitri,P. (2016) When chromatin organisation floats astray: the Srcap gene and Floating-Harbor syndrome. J Med Genet, 53, 793-797. 

Figurive

A

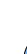

(

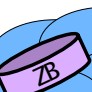

SWR1C
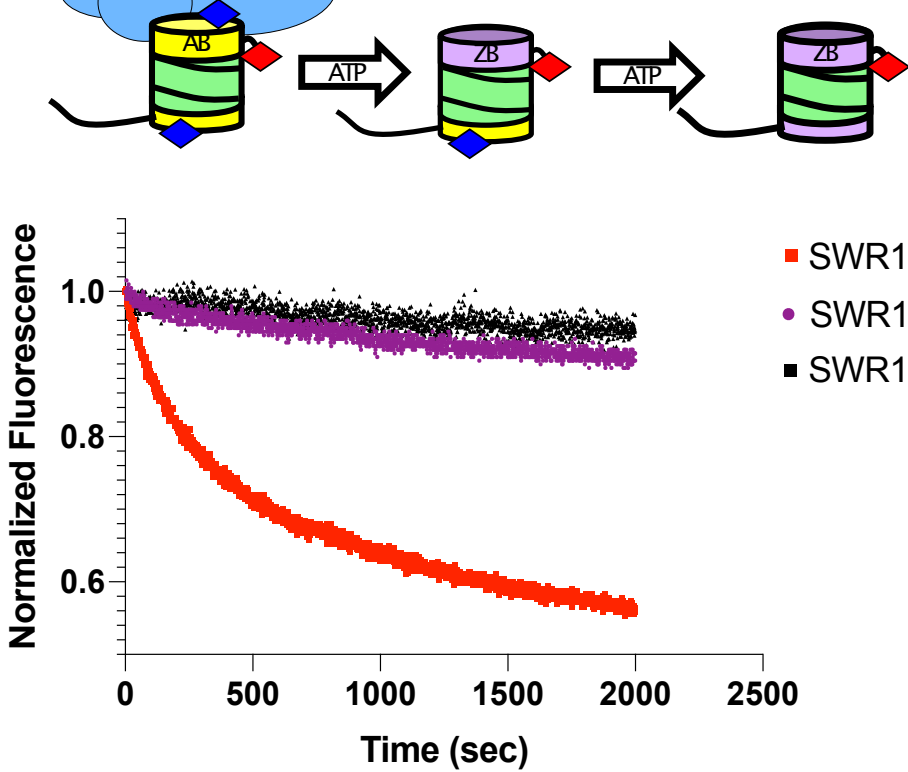

C

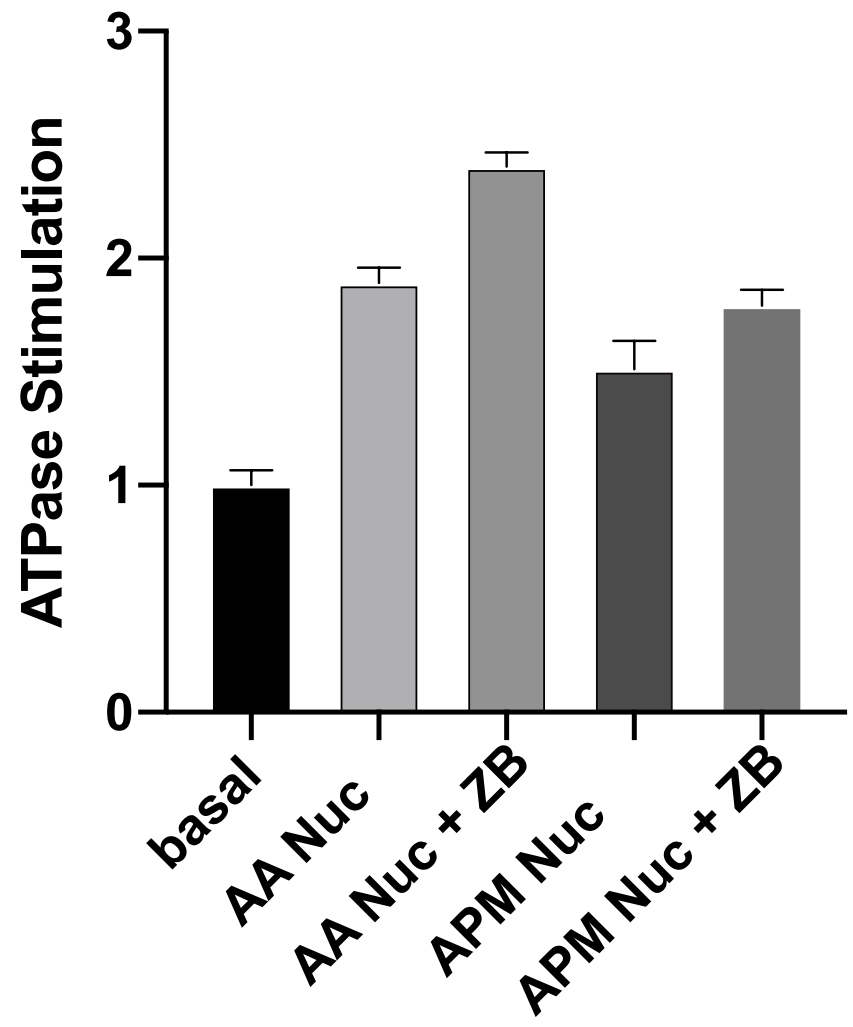

B

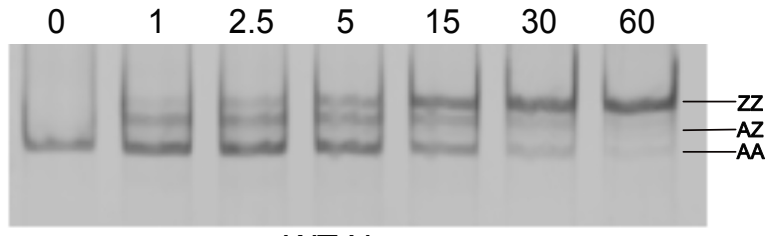

WT Nuc

- SWR1C WT nuc

- SWR1C APM nuc

- SWR1C ${ }^{\text {swc5s }}$ WT nuc

Time (min)
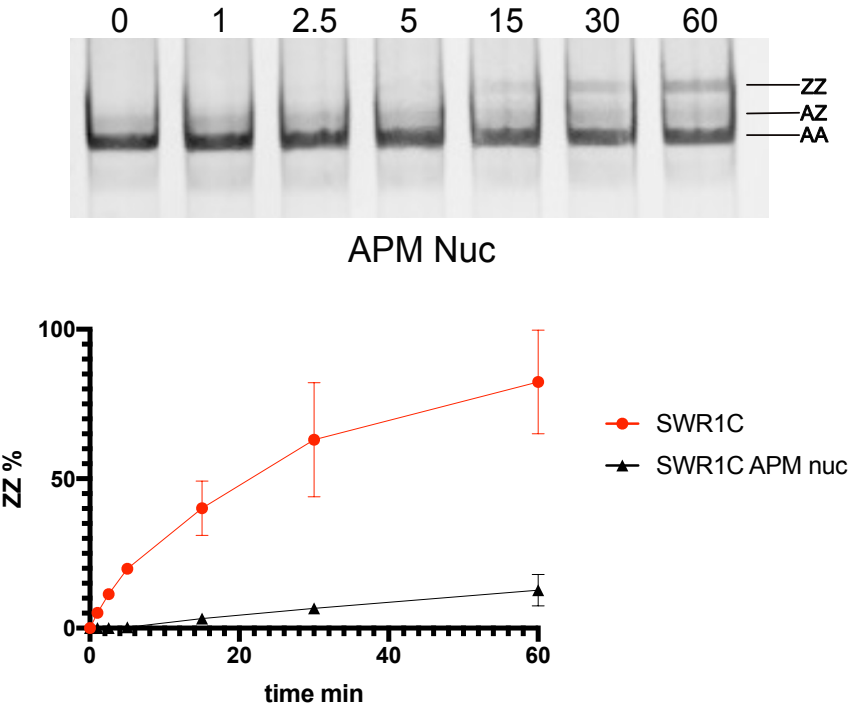

WT Nuc

APM Nuc
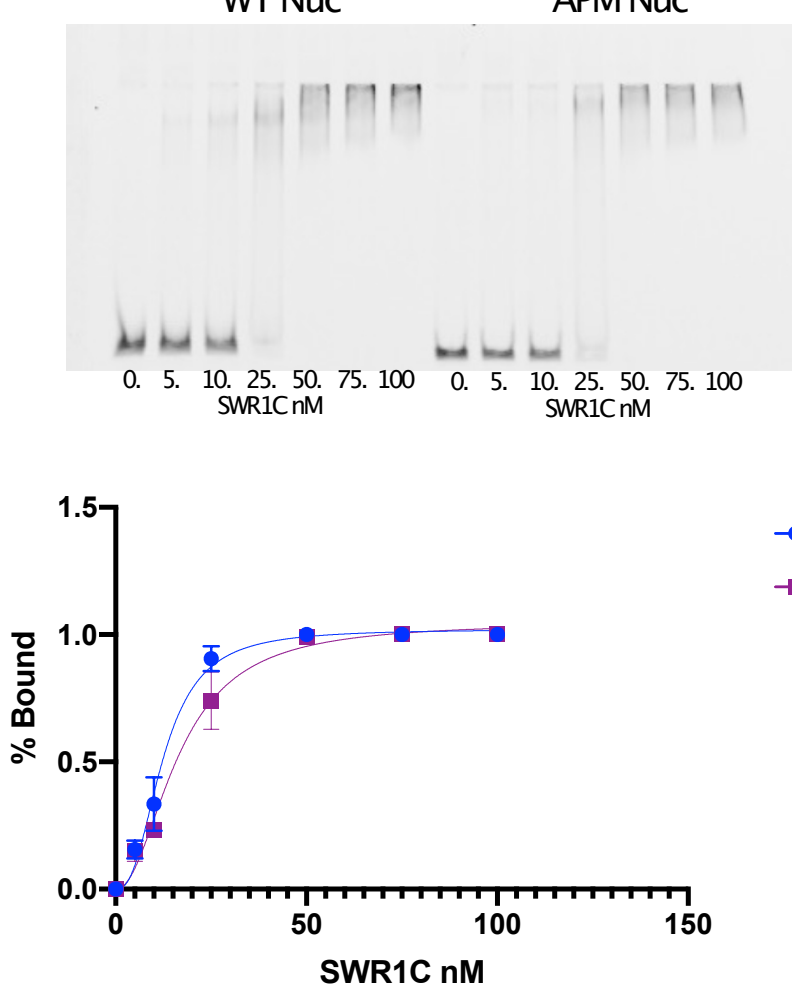

- WT Nuc

- APM Nuc 


\section{Figure 1}

The nucleosomal acidic is required for SWR1C catalyzed dimer exchange. (A) 77N0 FRET nucleosomes were remodeled by SWR1C with unlabeled H2A.Z/H2B dimers under single turnover conditions (30nM SWR1C, 10nM 77N0 nucleosomes, 70nM dimers, 1mM ATP). Dimer eviction is monitored by measuring the Cy5 emission at $670 \mathrm{~nm}$. SWR1C was able to catalyze the dimer exchange reaction on a WT 77N0 nucleosome (Red line), but SWR1C ${ }^{\mathrm{swc} 5 \Delta}$ on WT 77N0 (Black line) and SWR1C with APM 77N0 nucleosomes had no detectable dimer exchange activity. (B) SWR1C (30nM) dimer deposition was performed by measuring the incorporation of $\mathrm{H} 2 \mathrm{~A} . \mathrm{Z}^{3 \mathrm{xFlag}} / \mathrm{H} 2 \mathrm{~B}(70 \mathrm{nM})$ into $(10 \mathrm{nM})$ WT $77 \mathrm{~N} 0$ nucleosomes or APM $77 \mathrm{~N} 0$ nucleosomes. H2A. $Z^{3 \times F l a g} / \mathrm{H} 2 \mathrm{~B}$ incorporation was quantified and plotted below (WT red, APM black). (C) ATPase stimulation was measured using a phosphate sensor (1nM SWR1C, 15nM 77N0 nucleosome, 30nM H2A.Z/H2B dimers) or NADH coupled assay (20nM SWR1C, 75nM 77N0 nucleosome, 150nM H2A.Z/H2B dimers). ATPase stimulation was calculated by normalizing the basal rate of ATP hydrolysis by SWR1C to a value of 1 and measuring stimulation of ATPase activity in the presence of nucleosomes and dimers. (D) Increasing amounts of SWR1C was titrated to (5nM) WT or APM 77N0 nucleosomes to measure binding affinity. The \% Bound was calculated by quantifying the disappearance of the nucleosome band and plotting in the graph show below. 
Fin

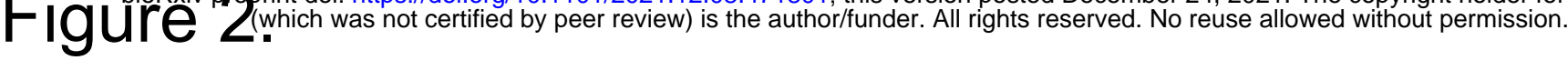

A

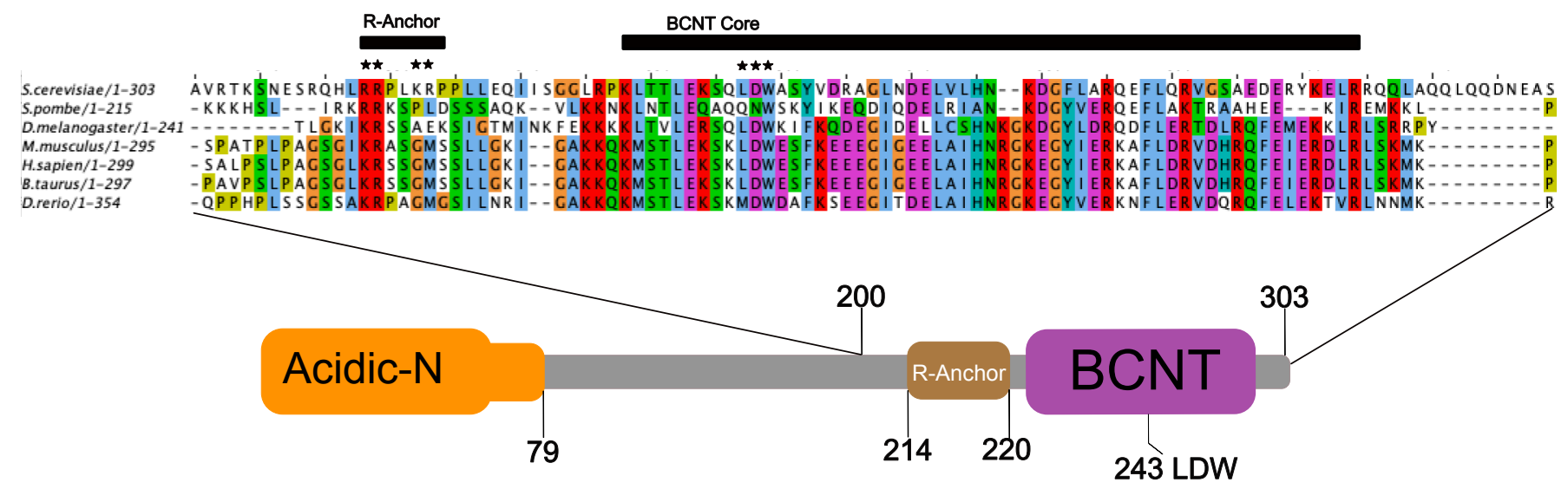

B

\section{Dimer Eviction Assay}
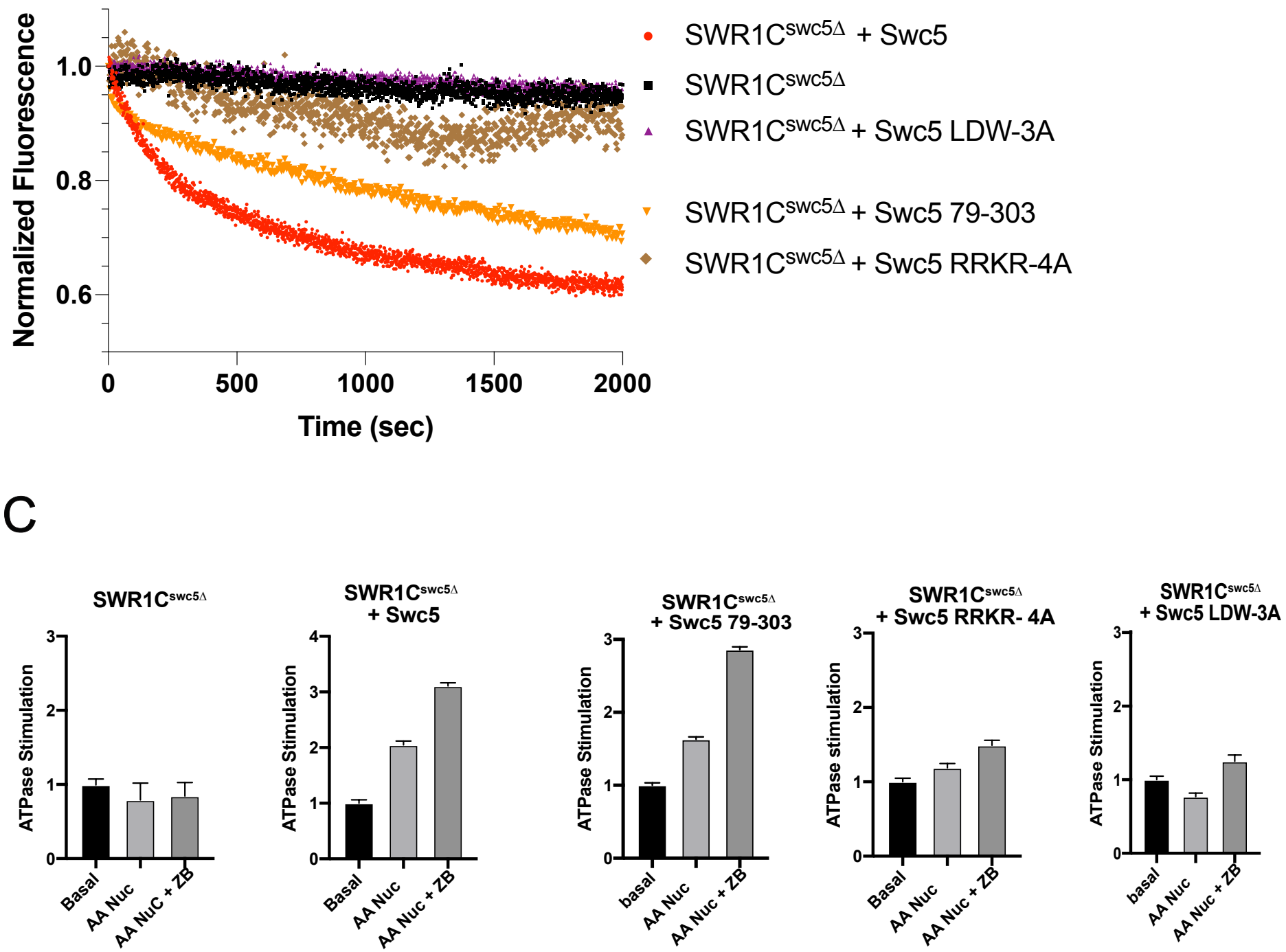


\section{Figure 2.}

Swc5 contains a conserved arginine anchor that is essential for dimer exchange and proper ATPase stimulation. (A) An alignment of Swc5 homologs revealed a conserved basic batch of residues upstream of the BCNT domain (asterisk denote residues that are mutated in biochemical assays). A schematic of the S.cerevisiae Swc5 protein highlights key regions. (B) Swc5 and Swc5 derivatives were reconstituted into SWR1C ${ }^{\text {swc5 } 5}$ complexes to measure their effect on dimer exchange using a FRET exchange assay (30nM SWR1C, 10nM 77N0, 70nM dimers). (C) ATPase stimulation was measured identically using either the phosphate sensor assay or NADH coupled assay. Basal ATPase activity of each SWR1C ${ }^{\mathrm{swc} 5 \Delta}$ complex was normalized to 1 and stimulation upon addition of nucleosomes and dimers was determined relative to basal activity. 
[ $\quad$ bioRxiv porint doi: https://doi.org/10.1101/2021.12.08.471801; this version posted December 24,2021 . The copyright holder for this preprint A

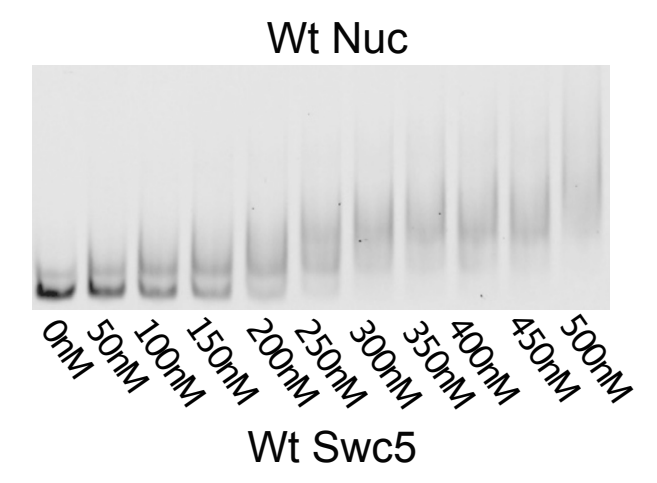

B

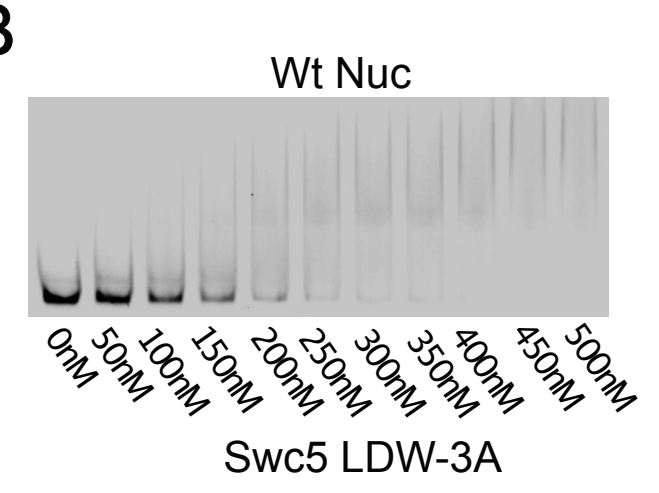

0

APM Nuc

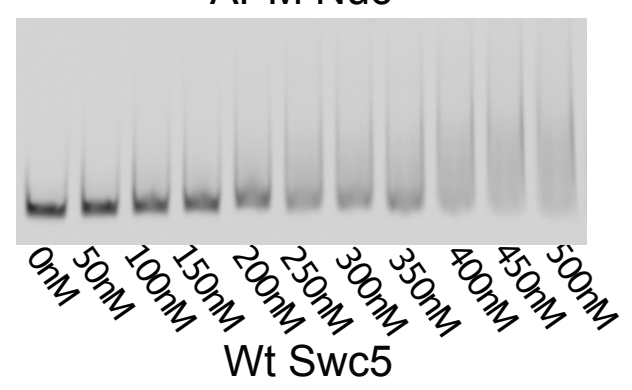

E

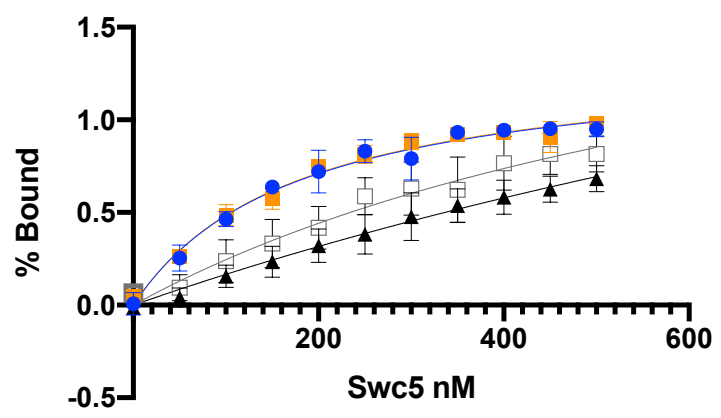

$\rightarrow$ Wt Swc5

-- Swc5 LDW->AAA

$\leftarrow$ Swc5 RRKR-AAAA

$\because$ Wt Swc5 APM nuc
D

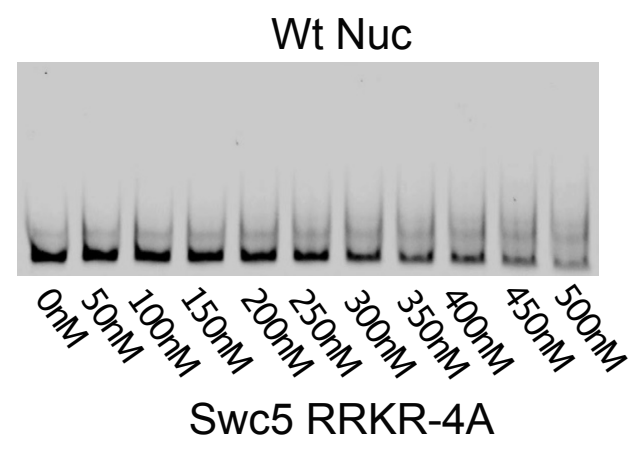

\begin{tabular}{|l|r|r|}
\hline One site specific binding & & \\
\hline & Kd(nM) & R squared \\
\hline Wt Swc5 & 178 & 0.9609 \\
\hline Swc5 DW & 176.7 & 0.9697 \\
\hline Swc5 RRKR & 1926 & 0.9035 \\
\hline Wt Swc5APM & 809.8 & 0.8639 \\
\hline
\end{tabular}




\section{Figure 3:}

Swc5 binding in vitro to nucleosomes requires the nucleosomal acidic patch and Swc5 arginine anchor residues. Gel mobility shift were performed with (A) WT Swc5 with 5nM 0N0 WT nucleosomes, (B) WT Swc5 with 5nM APM nucleosome, (C) Swc5 $5^{\text {LDW-3A }}$ with $5 \mathrm{nM}$ WT nucleosomes, and (D) Swc5 $5^{\text {RRKR-4A }}$ with WT nucleosomes. (E) The Kd for each condition was calculated by measuring the disappearance of the nucleosome band. 
Fivir - 101 (which was not certified by peer review) is the author/funder. All rights reserved. No reuse allowed without permission.

A

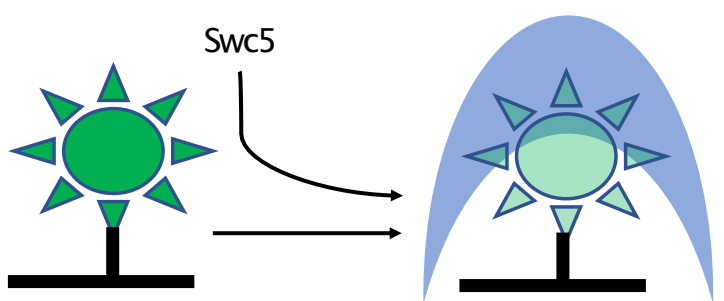

Oregon Green Probe

Fluorescence Quenching

H4 Tail or Acidic Patch

B

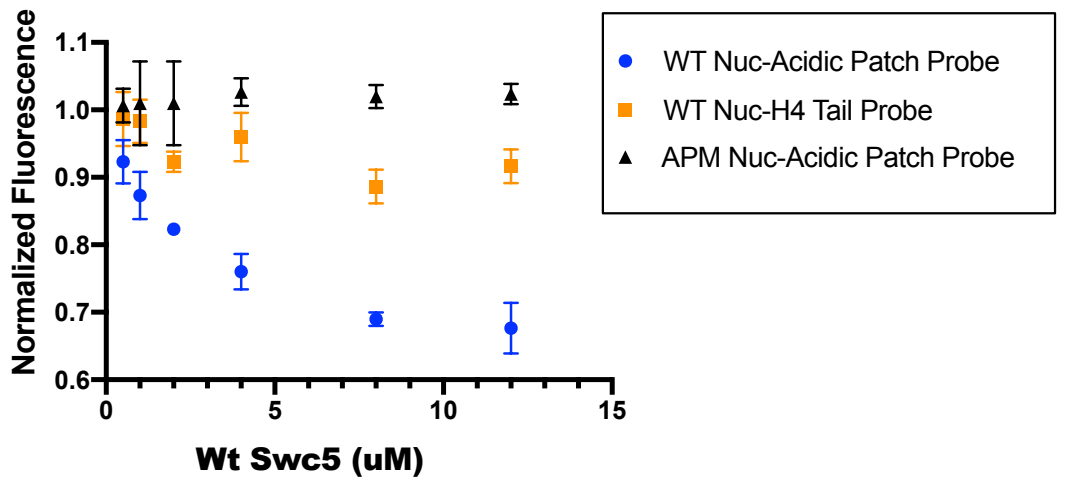

C

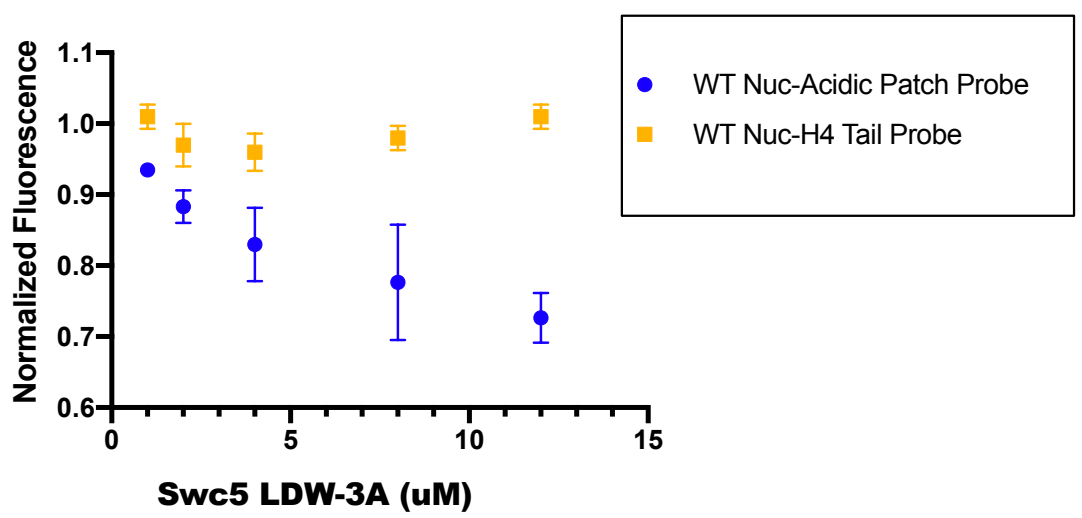

$\mathrm{D}$

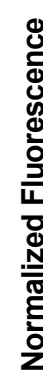
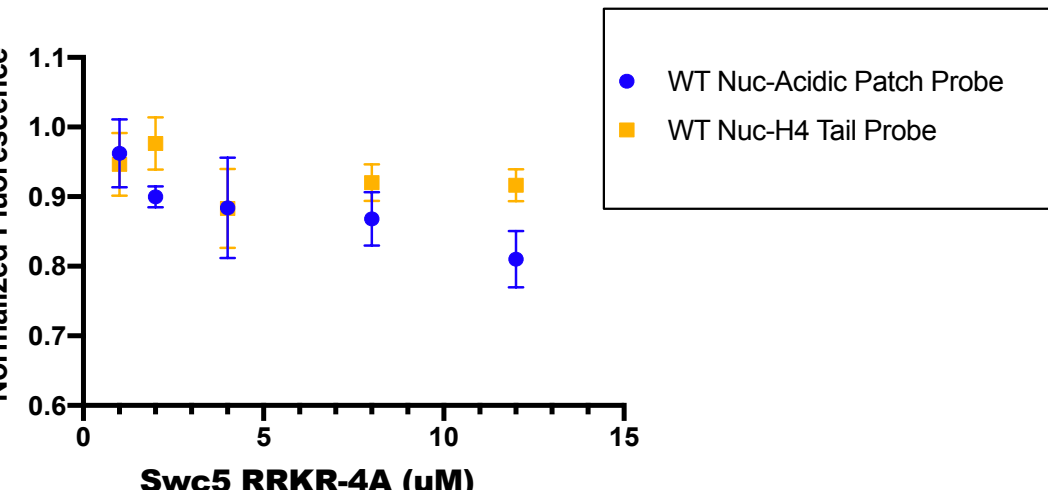


\section{Figure 4:}

Swc5 binding to nucleosomes is mediated by interaction between its arginine anchor and the nucleosomal acidic patch. (A) A schematic of fluorescent quenching using the Oregon Green fluorophore. Oregon Green fluorescence is sensitive to changes to its chemical environment resulting in a decrease in its fluorescence intensity. Oregon Green was covalently attached to the acidic patch (H2B S115C) or on the H4 histone tail (Q27C). (B) WT Swc5 was added to 10nM of WT 0N0 acidic patch probe nucleosomes (Blue), WT 0N0 H4 tail probe nucleosomes (Orange), or APM 0N0 acidic patch probe nucleosomes (Black). (C) Swc5 ${ }^{\text {LDW-3A }}$ showed the same binding pattern as its WT counterpart where it quenched WT acidic patch probe nucleosomes (Blue) and had no detectable quenching on $\mathrm{H} 4$ tail probe nucleosomes (Orange). (D) Swc5 ${ }^{\text {RRKR-4A }}$ was unable to quench the WT acidic patch probe nucleosome (Blue) and the $\mathrm{H} 4$ tail probe nucleosome. 
$F_{1}$

Figure $5^{\text {whi }}$

A

Control

10fold Serial Dilution

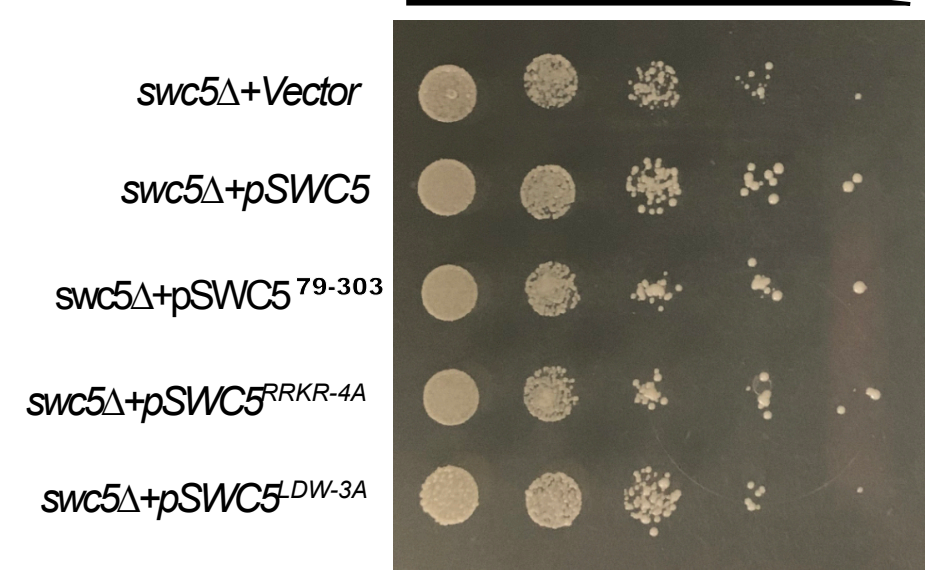

$3 \%$ Formamide

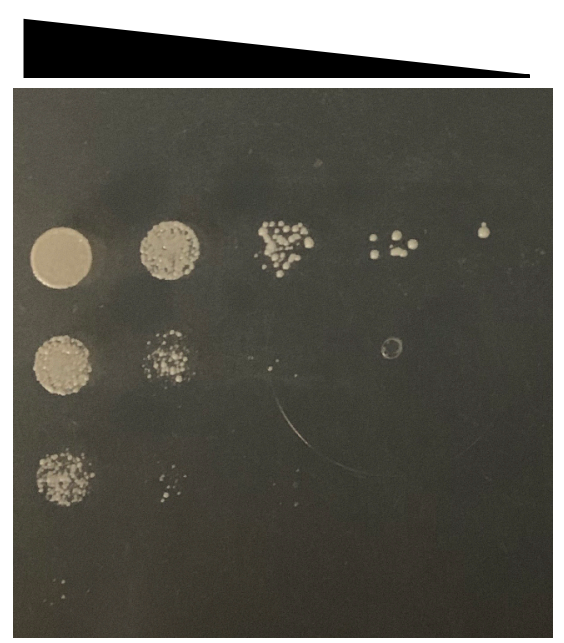




\section{Figure 5.}

Swc5 arginine anchor residues are important for SWR1C function in vivo. (A) Genetic complementation using a swc5 $\Delta$ haploid strain transformed with an empty pRS416 CEN ARS URA3 vector or this vector containing various alleles of SWC5. Cells were spotted onto plates with synthetic complete media lacking uracil with or without $3 \%$ formamide. 
bioRxiv preprint doi: https://doi.org/10.1101/2021.12.08.471801; this version posted December 24, 2021. The copyright holder for this preprint (which was not certified by peer review) is the author/funder. All rights reserved. No reuse allowed without permission.

Figure S1.

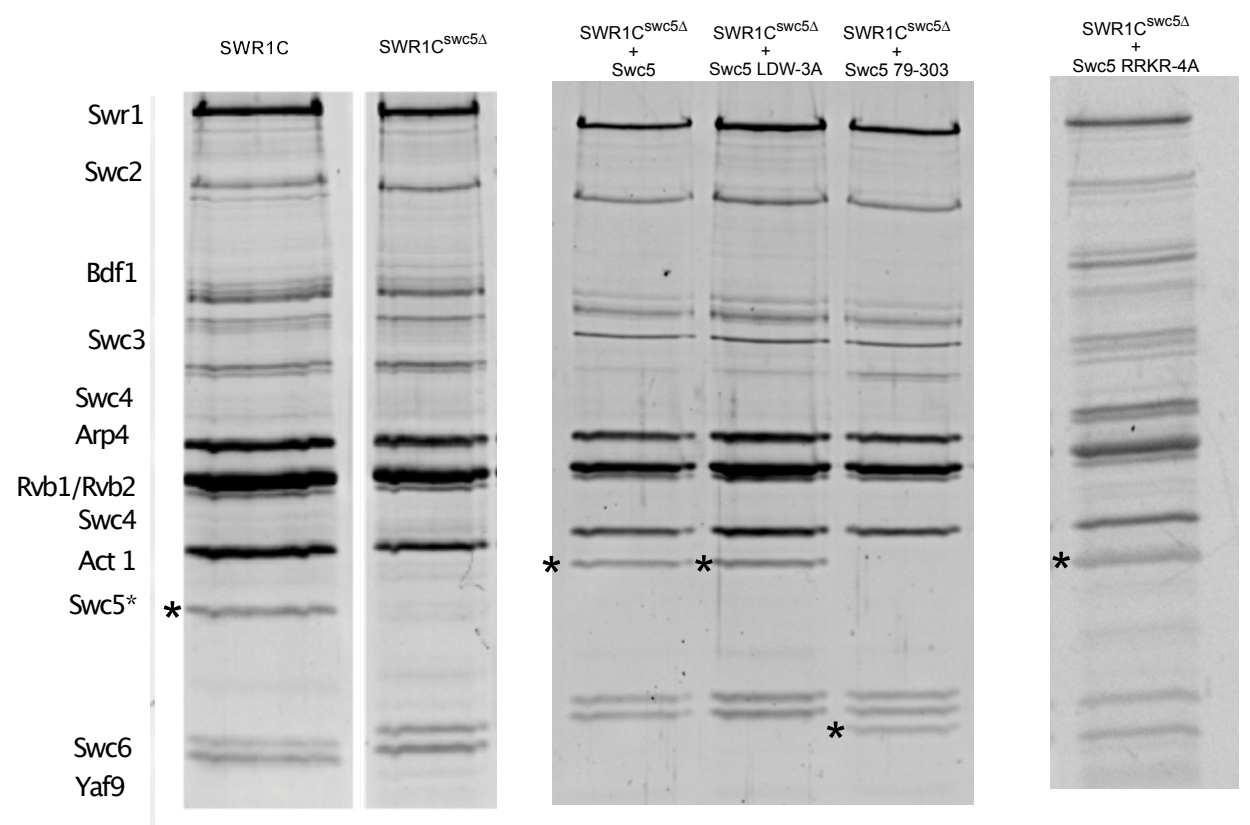


bioRxiv preprint doi: https://doi.org/10.1101/2021.12.08.471801; this version posted December 24, 2021. The copyright holder for this preprint (which was not certified by peer review) is the author/funder. All rights reserved. No reuse allowed without permission.

\section{Supplementary Figure S1.}

SWR1C complexes used in this study (A) SDS PAGE gels of SWR1C and SWR1C ${ }^{\text {swc5 }}$ complexes show stoichiometric amounts Swc5. The Swc5 band is denoted by an asterisk. 
bioRxiv preprint doi: https://doi.org/10.1101/2021.12.08.471801; this version posted December 24,2021 . The copyright holder for this preprint (which was not certified by peer review) is the author/funder. All rights reserved. No reuse allowed without permission.

Figure S2.

A

B
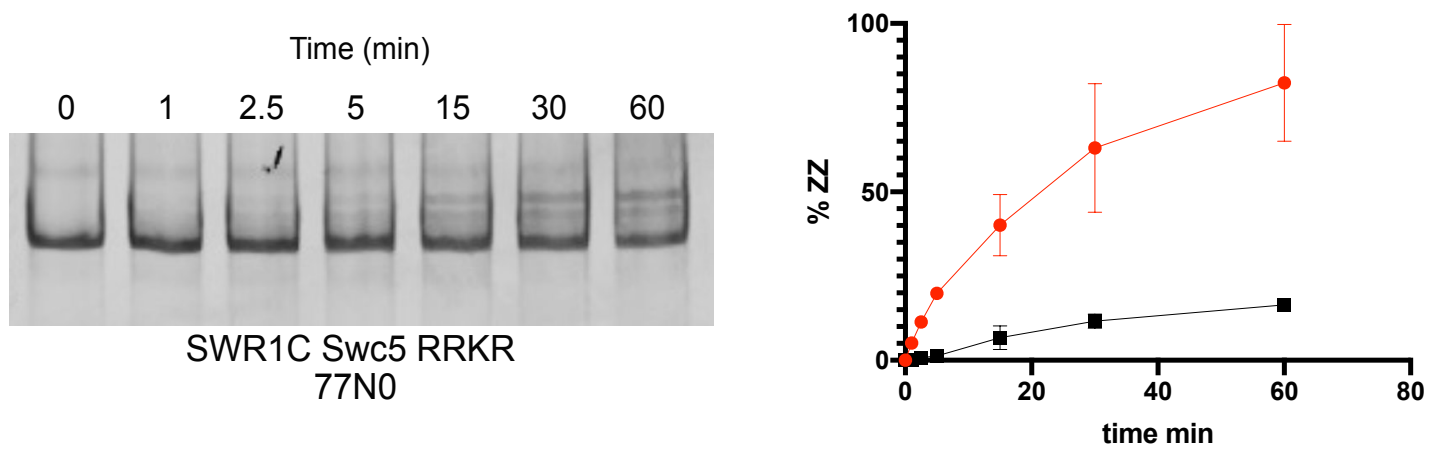

$\rightarrow$ SWR1C WT 77N0

$\rightarrow$ SWR $1 C^{\text {swc } 5 \Delta+\text { SWC5 }}$ RRKR-4A WT 77N0 


\section{Supplementary Figure S2.}

SWR1C $^{\text {swc5 }}+$ Swc5 $^{\text {RRKR-4A }}$ is defective for H2A.Z deposition. (A) Single turnover dimer deposition assay with (30nM) SWR1C $\mathrm{Swc}^{\mathrm{sw}}+\mathrm{Swc5}^{\mathrm{RRKR}-4 \mathrm{~A}},(10 \mathrm{nM}) 77 \mathrm{~N} 0$ nucleosome, and (70nM) H2A.Z/H2B ${ }^{3 x f l a g}$. Reaction was initiated with $1 \mathrm{mM}$ ATP. (B) Incorporation of H2A.Z $Z^{3 \times F l a g} / H 2 B$ by SWR $1 C^{\text {swc5 }}+$ Swc $5^{\text {RRKR-4A }}$ (Black line) was plotted and compared to the WT SWR1C using the same substrates (Red line). 


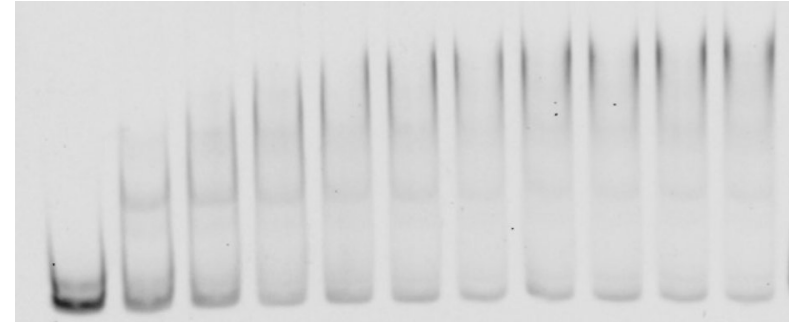

0. 20. 40. 60. 80.100120 .140 160. 180. 200. Swc5 nM

\section{B}

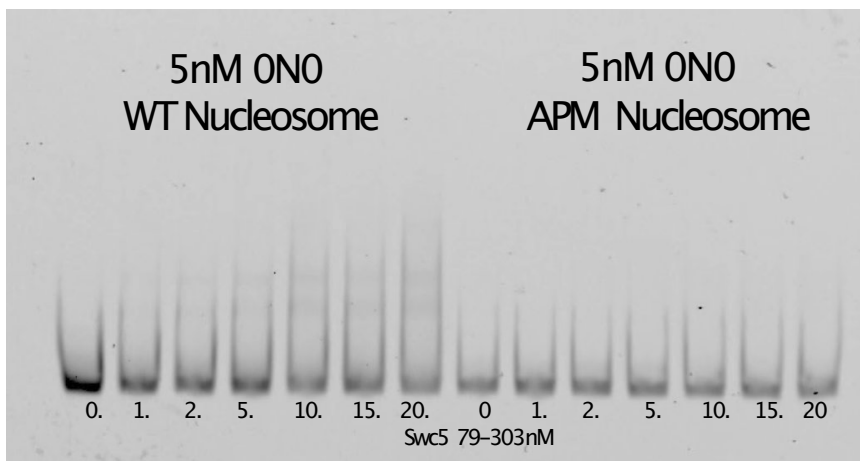

C

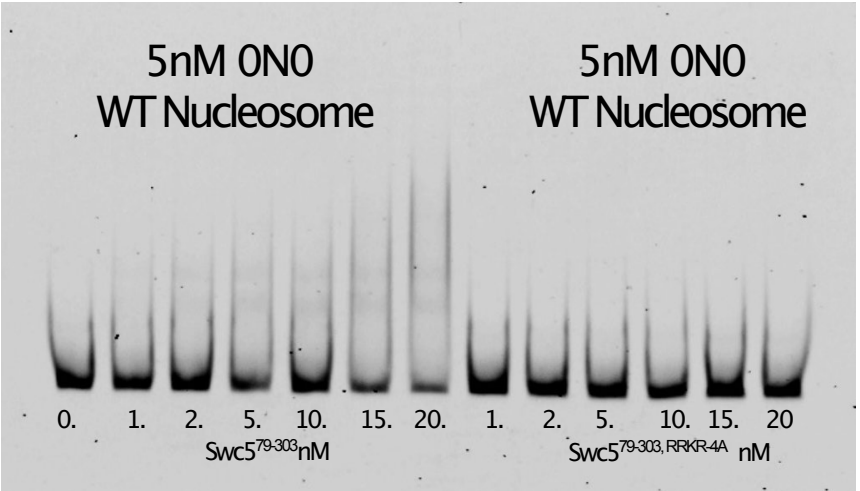


bioRxiv preprint doi: https://doi.org/10.1101/2021.12.08.471801; this version posted December 24, 2021. The copyright holder for this preprint (which was not certified by peer review) is the author/funder. All rights reserved. No reuse allowed without permission.

\section{Supplementary Figure S3.}

$\mathrm{Swc5}^{\text {79-303 }}$ requires the nucleosomal acidic patch and arginine anchor to bind nucleosomes.

(A) Gel shift binding assay with Swc5 $5^{79-303}$ and 5nM 0N0 nucleosomes (WT or APM) (B) Swc 5 ${ }^{79-303}$ and Swc5 $5^{79-303, R R K R-4 A}$ were added to 5nM WT 0N0 nucleosomes. 


\section{A}

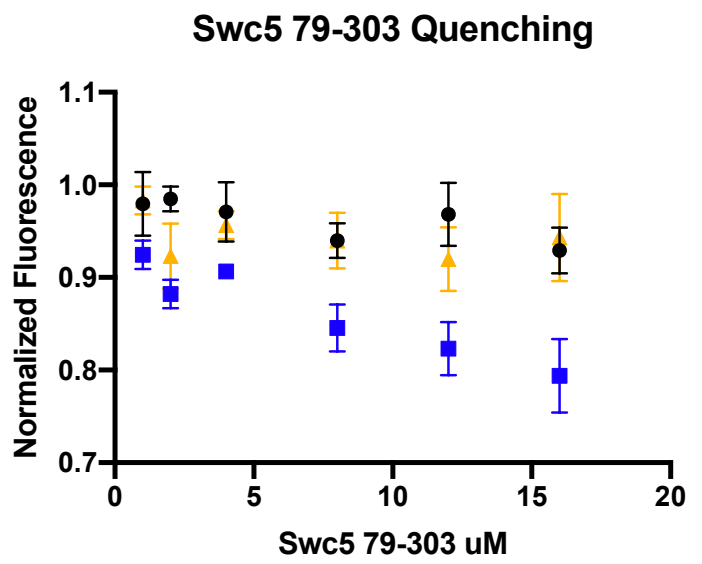

- APM Nucleosome Acidic Patch Probe Swc5 79-303

- WT Nucleosome Acidic Patch Probe Swc5 79-303

$\triangle$ WT Nucleosome Acidic Patch Probe Swc5 79-303 RRKR-4A 


\section{Supplementary Figure S4}

Swc5 ${ }^{79-303}$ specifically binds to nucleosome at the acidic patch. Fluorescent quenching was performed using a the Oregon Green fluorophore located on the acidic patch (H2B S115C) of 0N0 WT or APM nucleosome. 10nM nucleosomes incubated with increasing amounts of either Swc5 ${ }^{79-303}$ or Swc5 $5^{79-303, \text { RRKR-4A }}$. Swc5 $5^{79-303}$ quenched the Oregon Green probe on the acidic patch of WT 0N0 nucleosomes (Blue), but not the probe on the APM nucleosome (Black). Swc5 ${ }^{79-303, R R K R-4 A}$ was unable to quench the probe on the WT 0N0 nucleosome (Orange). 